\title{
Genome-wide comparison of four MRSA clinical isolates from Germany and Hungary
}

\author{
Romen Singh Naorem ${ }^{1}$, Jochen Blom ${ }^{2}$, Csaba Fekete ${ }^{\text {Corresp. } 1}$ \\ 1 Department of General and Environmental Microbiology, University of Pécs, Pécs, Hungary \\ 2 Bioinformatics \& Systems Biology, Justus-Liebig-Universität Gießen, Gießen, Germany \\ Corresponding Author: Csaba Fekete \\ Email address: fekete@gamma.ttk.pte.hu
}

Staphylococcus aureus is a drug-resistant pathogen, capable of colonizing diverse ecological niches and causing a broad spectrum of infections related to a community and healthcare. In this study, we choose four methicillin-resistant $S$. aureus (MRSA) clinical isolates from Germany and Hungary based on our previous polyphasic characterization finding. We assumed that the selected strains have a different genetic background in terms of the presence of resistance and virulence genes, prophages, plasmids, and secondary metabolite biosynthesis genes that may play a crucial role in niche adaptation and pathogenesis. To clarify these assumptions, we performed a comparative genome analysis of these strains and observed many differences in their genomic compositions. The Hungarian isolates (SA H27 and SA H32) with ST22-SCCmec type IVa have fewer genes for multiple-drug resistance, virulence, and prophages reported in Germany isolates. Germany isolate, SA G6 acquires aminoglycoside (ant(6)-la and aph(3')-III) and nucleoside (sat-4) resistance genes via phage transduction and may determine its pathogenic potential. The comparative genome study allowed the segregation of isolates of geographical origin and differentiation of the clinical isolates from the commensal isolates. This study suggested that Germany and Hungarian isolates are genetically diverse and showing variation among them due to the gain or loss of mobile genetic elements (MGEs). An interesting finding is the addition of SA G6 genome responsible for the drastic decline of the core/pan-genome ratio curve and causing the pan-genome to open wider. Functional characterizations revealed that $S$. aureus isolates survival are maintained by the amino acids catabolism and favor adaptation to growing in a protein-rich medium. The dispersible and singleton genes content of $S$. aureus genomes allows us to understand the genetic variation among the $\mathrm{CC} 5$ and $\mathrm{CC} 22$ groups. The strains with the same genetic background were clustered together which suggests that these strains are highly alike, however comparative genome analysis exposed that the acquisition of phage elements, and plasmids through the events of MGEs transfer contribute to differences in their phenotypic 
characters. This comparative genome analysis would improve the knowledge about the pathogenic $S$. aureus strain's characterization, and responsible for clinically important phenotypic differences among the $S$. aureus strains. 
1 Genome-wide comparison of four MRSA clinical isolates from Germany and Hungary

2 Romen Singh Naorem ${ }^{1}$, Jochen Blom ${ }^{2}$, and Csaba Fekete ${ }^{1}$

3 'Department of General and Environmental Microbiology, University of Pécs, Pécs, Hungary.

$4 \quad{ }^{2}$ Bioinformatics \& Systems Biology, Justus-Liebig-Universität Gießen, Gießen, Germany.

5 Correspondence: Csaba Fekete, Department of General and Environmental Microbiology,

6 University of Pécs, Ifjusag utja. 6, Pécs 7624, Hungary.

7 Phone: $+36-72503600$

8 Email: fekete@gamma.ttk.pte.hu

9 ORCID: 0000-0001-9430-4643

10 ORCID: 0000-0002-6562-6106

11

12

13

14

15

16

17

18

19

20

21

22

23

24

25

26

27 


\section{Abstract}

Staphylococcus aureus is a drug-resistant pathogen, capable of colonizing diverse ecological niches and causing a broad spectrum of infections related to a community and healthcare. In this study, we choose four methicillin-resistant $S$. aureus (MRSA) clinical isolates from Germany and Hungary based on our previous polyphasic characterization finding. We assumed that the selected strains have a different genetic background in terms of the presence of resistance and virulence genes, prophages, plasmids, and secondary metabolite biosynthesis genes that may play a crucial role in niche adaptation and pathogenesis. To clarify these assumptions, we performed a comparative genome analysis of these strains and observed many differences in their genomic compositions. The Hungarian isolates (SA H27 and SA H32) with ST22-SCCmec type IVa have fewer genes for multiple-drug resistance, virulence, and prophages reported in Germany isolates. Germany isolate, SA G6 acquires aminoglycoside (ant(6)-Ia and aph(3')-III) and nucleoside (sat-4) resistance genes via phage transduction and may determine its pathogenic potential. The comparative genome study allowed the segregation of isolates of geographical origin and differentiation of the clinical isolates from the commensal isolates. This study suggested that Germany and Hungarian isolates are genetically diverse and showing variation among them due to the gain or loss of mobile genetic elements (MGEs). An interesting finding is the addition of SA G6 genome responsible for the drastic decline of the core/pan-genome ratio curve and causing the pan-genome to open wider. Functional characterizations revealed that $S$. aureus isolates survival are maintained by the amino acids catabolism and favor adaptation to growing in a protein-rich medium. The dispersible and singleton genes content of $S$. aureus genomes allows us to understand the genetic variation among the CC5 and CC22 groups. The strains with the same genetic background were clustered together which suggests that these strains are highly alike, however comparative genome analysis exposed that the acquisition of phage elements, and plasmids through the events of MGEs transfer contribute to differences in their phenotypic characters. This comparative genome analysis would improve the knowledge about the pathogenic $S$. aureus strain's characterization, and responsible for clinically important phenotypic differences among the $S$. aureus strains.

Keywords: Staphylococcus aureus, whole-genome sequence, comparative analysis, functional annotation, pan-genome analysis, phylogenetic analysis 


\section{Introduction}

Staphylococcus aureus is a notorious nosocomial, and community-acquired pathogen (Chambers and Deleo, 2009). It has the capability of colonizing diverse ecological niches within its human host, including the skin, blood, respiratory tract, and nasal passages (Deleo et al., 2009) and causing diverse ranges of the hospital and community-acquired infections such as skin and soft tissue infections (SSI) for example, carbuncles, abscesses, styes, and impetigo and lifethreatening infections such as bacteremia, necrotizing pneumonia, osteomyelitis, endocarditis, and sepsis (Götz et al., 2006; Mottola et al., 2016). Methicillin-resistant S. aureus (MRSA) acquired a mobile genetic element called Staphylococcal chromosomal cassette mec (SCCmec) accompanied by methicillin resistance gene (mecA) (Zhang et al., 2012). The $\beta$-lactam insensitive protein, penicillin-binding protein (PBP2a) encoded by $m e c A$ gene reduces affinity to penicillin and $\beta$-lactam antibiotics including methicillin, oxacillin, cefoxitin, etc., and develop resistance toward the $\beta$-lactam antibiotics (Jansen et al., 2006; Mistry et al., 2016). MRSA acquires an arsenal of antibiotic resistance genes (ARGs) and virulence factor encoding genes (VFGs) through horizontal gene transfer (HGT) and recombination (Chan et al., 2011; Hughes et al., 2005).

MRSA can anchor and colonize on epithelial surfaces and produce biofilm (Goudarzi et al., 2017). The biofilm produced by MRSA strains encase its cells in the exopolysaccharide matrix reduces the activity of antibacterial agents and immune defense. The dispersal of bacterial cells from the biofilm can result in secondary site infections and leading infections worsen and difficult to eradicate (Lister and Horswill, 2014). Biofilm formation is a complex process that consists of an extracellular polymeric matrix (ECM) formation involving polysaccharide intercellular adhesin (PIA), protein-protein interactions, and the incorporation of extracellular DNA (eDNA) (O'Gara, 2007; Payne and Boles, 2016). The biofilm formation is determined by the $i c a A D B C$ gene cluster, responsible for PIA and capsular polysaccharide/adhesion synthesis (Chaieb et al., 2005). MRSA possesses adhesive matrix molecules that are encoded by elastin (ebps), laminin (eno), clumping factors A and B (clfA and clfB), fibronectin A and B ( $f n b A$ and $f n b B)$, collagen ( $c n a)$, fibrinogen $(f i b)$, bone sialoprotein ( $b b p)$, etc (Lindsay et al., 2006). These molecules are exported to the bacterial cell surface to enable adherence with host tissues, leading to play a role in pathogenesis (Mazmanian et al., 1999). 
S. aureus acquires an arsenal of ARGs and VFGs that are subjected to HGT and recombination (Chan et al., 2011; Hughes et al., 2005). Hospital-associated MRSA (HA-MRSA) is often associated with metastatic infections and significant morbidity and mortality (Gould, 2005). However, Community-associated MRSA (CA-MRSA) infections have seen a high increase in prevalence, posing a greater threat to the public (Morens and Fauci, 2013). The genomic plasticity of $S$. aureus has facilitated the development of hypervirulent and drug-resistant strains, result in challenging issues to antibiotic treatment and health concern. The classical techniques such as antibiotic susceptibility test (AST) patterns and molecular typing methods such as SCCmec-typing, Pulse-Field Gel Electrophoresis (PFGE), Multi-Locus Sequence Typing (MLST), Multi-locus variable-number tandem-repeat (VNTR) analysis (MLVA), S. aureus protein A (spa)-typing, accessory gene regulator ( $a g r)$-typing are widely used to detect and differentiate several MRSA strains, and helpful for identifying the risk factors associated with MRSA infection which support the establishment of adequate infection control programs (Zhang et al., 2012; Mistry et al., 2016). However, these methods are expensive and time-consuming, and have limitations in infection control and investigating the nosocomial transmission due to low resolution (Du et al., 2011). In this modern era, whole-genome sequence-based typing offers an excellent resolution in global and local epidemiologic investigations of pathogen outbreaks and offers further data mining activities essentially for ARGs and VFGs profiling (Köser et al., 2012). So, the Next Generation Sequencer (NGS) basedgenome sequencing technique has become an essential tool in the clinical microbiology arenas for comparative genomic analysis of several other species of the Staphylococcus genus in terms of the niche adaptation, combat antibiotics, and emergence of new virulent strains in real-time. In our preliminary study, the polyphasic characterization of 35 . aureus strains originated from Germany, and Hungary was performed. This characterization included antibiotic resistance test (ART), biochemical tests, biofilm-forming assay, and PCR based typing techniques involving the amplification of mecA, pvl, SCCmec-type, spa type, coa-HaeIII-RFLP, and biofilm-associated genes. Principal component analysis from polyphasic characterization data showed that the strains originated from the same geographical region were found in the close group while SA G8, Germany strain was grouped with other Hungarian strains (Naorem et al., 2020). The Hungarian strains (SA H27 and SA H32) belonged to the same Clonal Complex (ST22/SCCmec-IV) were clustered in the same group, however, these strains were isolated from the different sites of 
119 infections (nostrils and trachea) and showed different antibiotic resistance patterns and biofilm-

120 forming abilities. Similarly, the strains collected from Germany viz., SA G6, and SA G8

121 belonged to the same Clonal Complex (ST228/SCCmec-I and ST225/ SCCmec-II) having similar

122 antibiotic resistance pattern, and biofilm-forming profiles, but these strains were isolated from

123 the different site of infections (skin and other body sites) and not clustered in the same group

124 (Naorem et al., 2020). Based on this information, these four S. aureus strains were chosen for in-

125 depth comparative genome levels study to better understand the genomic differences among the

126 strains. We assumed that the selected strains have a different genetic background in terms of the

127 presence of ARGs, VFGs, prophages, plasmids, and secondary metabolite biosynthesis genes

128 that may play a crucial role in niche adaptation and pathogenesis. To clarify these assumptions,

129 we performed a comparative genome analysis of these four strains and observed many

130 differences in their genomic compositions.

\section{Materials \& Methods}

\section{Bacterial strains used in this study}

133 In this study, four S. aureus isolates collected from Germany (SA G6, and SA G8) and

134 Hungarian (SA H27, and SA H32) were used. Hungarian isolate, SA H27 was reported as a

135 strong biofilm producer among them (Naorem et al., 2020).

136 pH tolerance assay

137 S. aureus strains were cultured overnight at $37^{\circ} \mathrm{C}$ in tryptic soy broth (TSB) (DB, Germany).

138 The cell density (colony forming units, CFU) was adjusted to a final concentration of $\sim 10^{6}$

$139 \mathrm{CFU} / \mathrm{ml}$ in $\mathrm{pH} 4.5 \mathrm{TSB}$ and $\mathrm{pH} 9.5 \mathrm{TSB}$. Cell suspension $(200 \mu \mathrm{l})$ were loaded into the 96-well

140 flat-bottomed polystyrene microtiter plate (Costar 3599; Corning; USA). The plates were

141 incubated at $37^{\circ} \mathrm{C}$ for $24 \mathrm{~h}$ without shaking, then the growth was measured at $492 \mathrm{~nm}$

142 wavelength using a Multiskan Ex microtiter plate reader (Thermo Electron Corporation, USA).

143 The experiments were performed in triplicate and analyzed using GraphPad Prism 6 software

144 package (Graphpad Software Inc, San Diego, CA).

145 Genomic DNA isolation and sequencing

146 The genomic DNA was extracted using the GenElute ${ }^{\mathrm{TM}}$ Bacterial Genomic DNA Kit (Sigma,

147 USA) following the manufacturer instructions. The concentration and purity of genomic DNA 
148 was measured using dsDNA HS (High Sensitivity) Assay Kit in Qubit 3.0 fluorometer (Thermo

149 Fisher Scientific Inc., USA) and subsequently DNA quality was visualized by agarose gel 150 electrophoresis.

151 Genomic libraries were prepared by using the NEB Next Fast DNA Fragmentation and Library

152 Preparation Kit, developed for Ion Torrent (New England Biolabs) and used according to $200 \mathrm{bp}$ 153 protocol. After chemical fragmentation, DNA size selection was performed on precast 2\% E-Gel 154 Size Select Gel (Thermo Fisher Scientific Inc., USA). The quality of the libraries was verified 155 using Agilent high sensitivity DNA assay kit (Agilent Technologies Inc., USA) in Agilent 2100 156 Bioanalyzer System (Agilent Technologies Inc., USA). For the template preparation, Ion PGM 157 Hi-Q View OT2 Kit was used (Thermo Fisher Scientific Inc., USA). The template positive beads 158 were loaded on Ion 316v2 Chip and sequenced using Ion PGM Hi-Q View Sequencing Kit on 159 Ion Torrent PGM sequencer (Thermo Fisher Scientific Inc., USA).

\section{Genome assembly and annotation}

161 In-silico trimming of adapter and barcode sequences and data analysis were performed using 162 Torrent Suite 5.4.0 (Thermo Fisher Scientific Inc., USA) and the trimmed paired-end reads were

163

164

165

166

167

168

169

170

171

172

173

174

175

176

177 assembled by de novo assembler SPAdes 3.7.1 software with 21, 33, 55, 77, 99, $127 \mathrm{k}$-mer values (Nurk et al., 2013). The assembly-stats and quality of genome completeness for each strain were estimated using the web platform QUEST (Gurevich et al., 2013). For identifying the closely related strains, the genome assemblies were analyzed by the kmerFinder 2.0 webplatform (Larsen et al., 2014). The genome assembly was aligned against the reference genome for the contigs rearrangement using the 'Move Contigs' algorithm in Mauve 2.4.0 (Darling et al., 2010) and further, scaffolds were generated with reference genome/ genome of closely related strains predicted by kmerFinder 2.0 as a guide for alignment using the reference-based scaffolder MeDuSa (Bosi et al., 2015). Gene annotation of the genome assemblies was performed via the fully automated RAST (Rapid Annotation using Subsystem Technology) (Aziz et al., 2008) and PATRIC 3.5.7 (Pathosystems Resource Integration Center) (Wattam et al., 2013) pipelines using the reference genome.

\section{In-silico characterization of genome assemblies}

In-silico epidemiologic characterization of genome assemblies was performed using SCCmecFinder-1.2 for the identification of SCCmec types (Kaya et al., 2018), spaTyper 1.0 
178 (Bartels et al., 2014) for spa type, and MLST 1.8 (Larsen et al., 2012) for Multilocus Sequence

179 Type in a web-based server provided by the Center for Genomic Epidemiology

180 (https://cge.cbs.dtu.dk/services/). In-silico arg (accessory gene regulator)- typing was performed

181 using the primers described by Shopsin et al. (Shopsin et al. 2003) in in-silico PCR amplification

182 tools (Bikandi et al., 2004).

183 The genome assemblies were screened for plasmid replicon (rep) genes using PlasmidFinder 2.1

184 (Carattoli et al., 2014) with default parameters. The identified nonaligned contig or scaffold

185 associated with plasmid sequences were extracted and used for the identification of full-length

186 plasmid regions using PLSDB (Plasmid Database) version-2020-03-04 (Galata et al., 2018) with

187 search strategy Mash screen, and the default values were a maximum P-value of 0.1 and a

188 minimum identity of 0.99 (https://ccb-microbe.cs.uni-saarland.de/plsdb/). Identified plasmids

189 were compared with the closest reference plasmids using Easyfig version 2.2.3 (Sullivan et al.,

190 2011). The identification and annotation of prophage sequences were performed by screening the

191 genome assemblies using PHASTER (PHAge Search Tool Enhanced Release) (Arndt et al.,

192 2016), and identified template phages were classified for their lifestyles using PHACTS (Phage

193 Classification Tool Set) (McNair et al., 2012).

194 In-silico mining of candidate ARGs and VFGs were performed using CARD (Comprehensive

195 Antibiotic Resistance Database) version 3.0.8 in RGI (Resistance Gene Identifier) version 5.1.0

196 platform (https://card.mcmaster.ca/analyze/rgi) (Alcock et al., 2020), and a comprehensive set of

197 S. aureus VFGs was analyzed using VFDB (Virulence Factor Database) in VFanalyzer (Liu et

198 al., 2019) and the PATRIC tool version 3.6.3 (https:/www.patricbrc.org/) (Wattam et al., 2013).

199 Further, heatmap and hierarchical clustering were generated to visualize the presence and

200 absence of VFGS and ARGs in S. aureus strains using a web-based application, Morpheus,

201 (https://software.broadinstitute.org/morpheus). Secondary metabolite biosynthesis gene clusters

202 and the detection of genes encoding bacteriocins were analyzed using antiSMASH 5.0 (Blin et

203 al., 2019) and BAGEL4 (Van Heel et al., 2018). The prediction of chromosomal genomic islands

204 was predicted by using IslandViewer 4 (Bertelli et al., 2017).

205 Comparative genomic analysis

206 The ANI was determined based on BLAST+ using the JSpeciesWS webserver (Richter et al.,

207 2016). The pairwise comparisons between the genomes of $S$. aureus isolates and their nearest 
208 reference genomes were conducted using GBDP (Genome BLAST Distance Phylogeny) under

209 the algorithm trimming and distance formula d5, and calculated each distance with 100 replicates

210 (Meier-Kolthoff et al., 2013). dDDH (Digital DNA-DNA Hybridization) values and confidence

211 intervals were calculated using the recommended settings of the GGDC 2.1 (Meier-Kolthoff et

212 al., 2013).

213 Genomes of $S$. aureus isolates and their reference strains were compared with CGViewer

214 (Circular Genome Viewer) server (Grant et al., 2008). The functional annotation was performed

215 using EggNOG (Evolutionary Genealogy of Genes: Non-supervised Orthologous Groups)

216 mapper 5.0 database (Huerta-Cepas et al., 2019) and RAST server-based SEED viewer

217 (Overbeek et al., 2014).

218 The pan-genome, core-genome, and singletons were calculated using four study genomes of $S$.

219 aureus isolates in EDGAR version 2.0 software framework (Blom et al., 2016). This pan-

220 genome analysis was extended using four study genomes coupled with three reference genomes

221 of $S$. aureus strains. The core-genome was analyzed in the genomes set using reciprocal best

222 BLAST hits of all CDS using EDGAR version 2.0 software framework (Blom et al., 2016). The

223 singletons were calculated for the contig of a strain by comparing to the CDS of a set of contigs

224 in EDGAR. The CDS that has no match with SRV (Score Ratio Value Plots) higher or equal the

225 master cut-off in any of the contigs were considered as singletons. The development of pan-

226 genome and core-genome sizes was analyzed using the core/pan development feature and as

227 well, the pan vs. core development plot was generated in EDGAR. Heap's Law function was

228 applied to calculate whether the pan-genome open or closed using the equation $\mathrm{n}^{\prime} k^{*} \mathrm{~N}^{\wedge}(-\alpha)$

229 where $\mathrm{n}=$ expected a number of genes; $\mathrm{N}=$ number of genomes; $k$ and $\alpha(\alpha=1-\gamma)$ are

230 proportionality constant and exponent, respectively (Tettelin et al., 2008). Heap's law predicted

231 that closed pan-genome (when $\alpha>1(\gamma<0)$ ), and open pan-genome (when $\alpha<1(0<\gamma<1)$ ).

232 According to Tettelin et al., 2008, core-genome and singletons developments were calculated by

233 the least-square fitting of exponential decay functions.

234 The Rcp (ratio of core-genome to that of pan-genome) was calculated (Ghatak et al., 2016).

235 Then, genomic subsets, including the number of core-genome and singletons in the gene pool,

236 were extracted, and flowerplot was drawn using in-house R scripts.

\section{Phylogenetic analysis}


238 The genome assemblies of the isolates were used for a whole genome-based phylogeny analysis

239 using TYGS (Type/Strain Genome Server) (Meier-Kolthoff and Göker, 2019) engaging with

240 genomes of closely related strains of $S$. aureus. The phylogenomic trees were reconstructed

241 using FastME 2.1.6.1 (Lefort et al., 2015) from the GBDP (Genome BLAST Distance

242 Phylogeny) distances calculated from genome sequences under the algorithm 'coverage' and

243 distance formula d5 (Meier-Kolthoff et al., 2013). The trees were rooted at the midpoint (Farris,

244 1972); branch supports were inferred from 100 pseudo-bootstrap replicates and visualized with

245 Interative Tool Of Life v4 (iTOL) (Letunic and Bork, 2019). The core SNPs of genome

246 sequences were extracted using Panseq (Laing et al., 2010) and the phylogenetic tree was

247 constructed using PhyML+SMS module in NGPhylogeny.fr (Lemoine et al., 2019) to select the

248 best evolutionary model, further the tree was annotated in Interative Tool Of Life v4 (iTOL)

249 (Letunic and Bork, 2019).

250 Results

251 The $S$. aureus isolates could survive at pH 4.5 through $\mathrm{pH} 9.5$ conditions with a survival rate of $\sim$

$25245 \%-84 \%$. SA G8 isolate showed the highest cell survival rate of $84.4 \%$ at acidic $\mathrm{pH}$ but its cell

253 survival rate drops down by $7 \%$ when subjected to alkaline $\mathrm{pH}$ conditions (Table S1).

\section{General genomic features of $\boldsymbol{S}$. aureus isolates}

255 The genomic DNA of S. aureus isolates was successfully sequenced in the IonTorrent PGM

256 sequencing platform. The average raw reads obtained from the genome sequencing of SA G6,

257 SA G8, SA H27, and SA H32 are 88.9, 69.6, 128.3, and 92.7 million bases $(\mathrm{Mb})$ for genomes

258 of SA G6, SA G8, SA H27, and SA H32 strains respectively. The closely related strains

259 identified by kmerFinder 2.0 were S. aureus subsp. aureus ST228 (HE579073), S. aureus subsp.

260 aureus JH9 (CP000703) for SA G6 and SA G8 strains, respectively. Also, S. aureus subsp.

261 aureus HO 50960412 (HE681097.1) was identified closely related strains for SA H27 and SA

262 H32 strains. Among the S. aureus isolates, SA G8 has the largest genome size (28633393 bp)

263 with high \% GC content (32.81\%). The numbers of protein-coding sequences (CDSs) in the $S$.

264 aureus strains varied from 2630 (SA H27) to 2743 (SA G8). The comparison of draft genome

265 assemblies, genome annotation, molecular typing, plasmid, and prophage features for S. aureus

266 genomes were summarized in Table $\mathbf{1 .}$ 


\section{Genes encoding plasmids}

268 The putative plasmids were detected in nonaligned contigs or scaffolds that exhibited an

269

270

271

272

273

274

275

276

277

278

279

280

281

282

283

284

285

286

287

288

289

290

291

292

293

294

295

296

297 unexpected high coverage level after the genome assemblies. A putative plasmid (p1G6) of 13331 bp length was identified at Scaffold 4 of the SA G6 genome consisting of the replication gene (repA). The p1G6 plasmid has $30.97 \%$ sequence coverage with plasmids pTW20_1 (FN433597.1) (Fig. 1A). The sequence coverage region of p1G6 with pTW20_1 constitutes the genes that encode for proteins such as IS6 family transposase, replication-associated protein (Rep), cadmium resistance transporter (CadD), cadmium efflux system accessory protein $(\mathrm{CadX})$, replication initiation protein A (RepA), quaternary ammonium compound efflux MFS transporter (QacA), multidrug-binding transcriptional regulator (QacR), DUF536 domaincontaining protein $(\mathrm{mP})$, AAA family ATPase (Abp), hypothetical proteins, HAD hydrolase family protein, and IS257 family transposase. The SA H32 genome also consists of a putative plasmid (p2H32) having a length of $2530 \mathrm{bp}$ located at Scaffold 3 and showed $71.32 \%$ sequence coverage with plasmids AR_0472 (NZ_CP029648.1). It consists of a replication gene (repL) and carried an erythromycin resistance gene $(e m r C)$ (Fig. 1B). The identified plasmids of $S$. aureus encode no other factors for their transfer, such plasmids may transfer via phage transduction (McCarthy and Lindsay, 2012). The linear graphical map of plasmid comparison was represented in Fig. 1.

\section{Characteristic of prophages-like elements}

The genomes of $S$. aureus isolates have several prophages and phage-like element regions and these prophages were belonged to the Siphoviridae family and having temperate lifestyles. The highest number of prophage regions was found in the genome of SA G8 isolate including three intact prophages (phiG8.2, phiG8.3, and phiG8.4), a questionable (phiG8.1), and an incomplete (phiG8.5) prophages. Four prophage regions were found in the genome of SA G6 isolate including an intact prophage (phiG6.3), two questionable prophages (phiG6.1 and phiG6.4), and an incomplete prophage (phiG6.2). The genome of SA H27 isolate harbor three intact prophages (phiH27.1, phiH27.2, and phiH27.3) while the genome of SA H32 harbor only one intact prophage (phiH32.1). The lukF-PV and $l u k M$ genes (Bicomponent leukotoxins), and plc gene (Phospholipase C) were identified in the prophages of phiG6.4, phiG8.4, phiH27.2, and phiH32.1. The prophages of phiG6.3, phiG8.4, and phiH27.2 carried sak gene (staphylokinase) and scn gene (staphylococcal complement inhibitor). Chemotaxis inhibitory protein encoded by 
298

299

300

301

302

303

304

305

306

307

308

309

310

311

312

313

314

315

316

317

318

319

320

321

322

323

324

325

326

chp gene was identified in phiG8.4 and phiH27.2 prophages. Enterotoxin A encoded by sea gene was harbored by the prophages of phiG6.3 and phiG8.4. Hemolysin genes such as $h l b$ ( $\beta$ hemolysin), and $h \lg B$ ( $\square$-hemolysin B) were found in the prophages of phiH27.2, and phiH32.1. In addition to virulence factors, phiG6.4 prophage carried ARGs genes that conferred resistance to beta-lactamase (blaZ), aminoglycoside (ant(6)-Ia and aph(3')-III) and nucleoside (sat-4) antibiotics. The comparative analysis of VFGs associated with putative prophages was summarized in Table S2.

\section{In-silico analysis of antimicrobial resistance and associated genes in the genomes}

Four study genomes of $S$. aureus isolates shared 63.3\% (19/30) of antibiotic resistance and associated genes (Fig. 2). The shared genes comprise methicillin-resistant PBP2a (mec $A$ and mecRl); multidrug resistance efflux (ygaD); fluoroquinolone (nor $A$ and gyrA); fluoroquinolone and acridine dye ( $\operatorname{arl} S$ and $\operatorname{arlR})$; glycylcycline ( penam, cephalosporin, glycylcycline, rifamycin, phenicol, triclosan, fluoroquinolone ( $m g r A$ and marR); lipopeptide ( $p g s A, c l s A$ and $r p o C)$; rifampicin ( $r p o B)$; aminocoumarin ( $g y r B$ and parE); dihydrofolate reductase ( $d f r A /$ folA $)$ and defensin ( $m p r F / f m t C$, multiple peptide resistance factor) that play roles in resistance mechanism including antibiotic efflux, antibiotic target alteration, and antibiotic target replacement. The comparative analysis of ARGs revealed that the genome of SA G6 isolate acquired additional ARGs responsible for the resistance of aminoglycoside

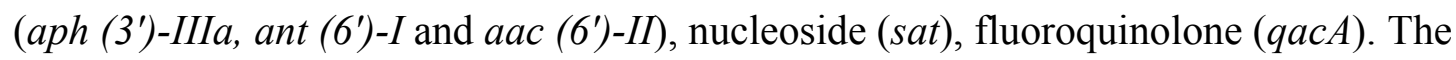
macrolide, lincosamide, streptogramin (MLS) erythromycin antibiotic resistance genes (emrA) were detected in the genomes of SA G6 and SA G8 isolates while the genome of SA H32 isolate present emrC gene. The penicillin resistance gene (blaZ) was found absent in SA G8 isolate.

This in-silico identification and our previous antibiotic susceptibility test results were correlated with beta-lactam, erythromycin (MLS), and vancomycin antibiotic resistance analysis (Naorem, et al., 2020). The secondary metabolite biosynthetic gene clusters identified among the genomes were staphylobactin, aureusimine, bacteriocin, and staphyloferrin A. The auto-inducing peptide (AIP)-II gene was identified in SA G6 and SA G8 genomes while AIP-I gene was identified in SA H27 and SA H32 genomes.

\section{In-silico analysis of virulence-factors encoding genes in the genomes}


327 The VFGs predicated against the VFDB revealed 59 VFGs were shared in all strains that are

328 responsible for adherence, toxin, anti-phagocytosis immune evasion, secretion system,

329 exoenzyme activity, and iron uptake (Fig. 3). The genome of SA G8 isolate has occupied 3.40\%

330 of VFGs against its CDS, whereas the genome of SA H32 isolate has $2.97 \%$ of VFGs against its

331 CDS.

332 Adherence associated genes shared in all genomes of $S$. aureus isolates were $63.63 \%(14 / 22)$

333 such as autolysin ( $a t l)$, cell wall-associated fibronectin-binding protein (ebh), elastin binding

334 protein $(e b p)$, fibrinogen binding protein $(e f b)$, fibronectin-binding proteins $\mathrm{A}(f n b A)$,

335 intercellular adhesin (icaA, icaB, icaC, icaD, icaR), ser-Asp rich fibrinogen-binding proteins

336 ( $s d r C, s d r D$, sdrE), staphylococcal protein A (spa) (Fig. 3A). The genome of SA G8 isolates

337 present $77.27 \%(17 / 22)$ of adherence associated genes with additional genes of clumping factor

338 A (clfA), clumping factor B (clfB), and fibronectin-binding proteins $(f n b B)$.

339 Type VII secretion system involves in membrane-associated proteins (esaA, ess A, ess $B$, and

$340 e s s C$ ), soluble cytosolic (esaB, esaE, esaG), and secreted virulence factors (esxA, esxB, esxC,

$341 e s x D$, and esaD) were identified in the genomes of SAG6 and SA G8 isolates while the SAH27

342 and SAH32 isolates absence esaD, esaE, esxB, esxC, and esxD genes (Fig. 3A).

343 The genomes of all isolates shared 29.41\% (10/34) of toxin genes such as alpha-hemolysin gene

$344(h l a)$, beta-hemolysin gene $(h l b)$, delta hemolysin gene $(h l d)$, gamma hemolysin A $(h \lg A)$,

345 gamma hemolysin B $(h \lg B)$, gamma hemolysin $\mathrm{C}(h \lg C)$, enterotoxin-like $\mathrm{O}($ selo), exfoliative

346 toxin type A (eta), and exotoxin (set13, set15) (Fig. 3B). The highest number of toxin genes

347 were identified in the genome of SA G8 i.e., 73.52\% (25/34), and in addition to shared genes, the

348 extra genes were enterotoxin A ( sea), enterotoxin B (seg), enterotoxin Yent1 (yent1),

349 enterotoxin-like K (selk), enterotoxin-like M (selm), enterotoxin-like N (seln), exotoxin (set6,

350 set7, set9, set11, set34, set37, set39), leukotoxin D (lukD), Panton-Valentine leukocidin (lukF-

$351 \quad P V)$.

352 The genes involve in anti-phagocytosis namely capsular polysaccharide synthesis genes belong

353 to stereotype 5 and 8 predominantly present in all the genomes of isolates were capsular

354 polysaccharide synthesis enzyme Cap5A (cap8A), capsular polysaccharide synthesis enzyme

355 Cap5B (cap8B), capsular polysaccharide synthesis enzyme Cap5C (cap8C), probable

356 polysaccharide biosynthesis protein $\mathrm{EpsC}$ (cap8D), capsular polysaccharide synthesis enzyme

357 Cap8E (cap8E), capsular polysaccharide synthesis enzyme Cap5F (cap8F), UDP-N-acetyl-L- 
358 fucosamine synthase (cap8G), capsular polysaccharide synthesis enzyme Cap5L (cap8L),

359 capsular polysaccharide synthesis enzyme Cap8M (cap8M), capsular polysaccharide synthesis 360 enzyme Cap8N (cap8N), UDP-N-acetyl-D-mannosamine dehydrogenase (cap8O) and UDP-N-

361 acetylglucosamine 2-epimerase (cap8P) (Fig. 3C). Other genes responsible for the host immune

362 evasion such as IgG-binding protein ( $\mathrm{sbi}$ ), staphylococcal complement inhibitor ( $\mathrm{scn}$ ), and 363 chemotaxis inhibiting protein ( $\mathrm{chp}$ ) were identified in all isolates.

364 Several exoenzymes encoding genes namely cysteine protease/ staphopain ( $\operatorname{ssp} B, \operatorname{ssp} C)$,

365 hyaluronate lyase (hysA), lipase (geh, lip) serine V8 protease (sspa), staphylocoagulase (coa),

366 staphylokinase (sak), and thermonuclease (nuc) were present in the genomes of all isolates.

367 However, five genes cluster for serine protease ( $s p l A, s p l B, s p l C, s p l D, s p l F)$ were absent in the 368 genomes of SA H27 and SA H32 isolates (Fig. 3C).

369 Eight genes involved in iron uptake mechanism including cell surface protein $(i s d A)$, cell surface 370 receptor $(i s d B)$ and cell wall anchor proteins (isdC), heme transporter component (isdD), high-

371 affinity heme uptake system protein (isdE), heme-iron transport system permease protein (isdF),

372 sortase B ( $\operatorname{srtB})$, heme-degrading monooxygenase; staphylobilin-producing (isd $G$ ) were

373 identified in all the genomes of isolates.

\section{Comparative genome analysis}

375 The genome comparative analysis based on ANIb matrices results indicated that the genome of 376 SA G8 isolate exhibits the nearest identities to all genomes. SA G8 genome showed $\sim 99.5 \%$ 377 identities to SA G6 genome. The genomes of SA G6 and SA G8 exhibited $\sim 99.7 \%$ identities to 378 S. aureus subsp. aureus ST228 (HE579071.1). Also, the genomes of SA H27 and SA H32 379 showed $99.9 \%$ identities to each other and these two genomes displayed the highest identities 380 (99.9\%) to S. aureus subsp. aureus HO 50960412 (HE681097.1) (Fig. S1). The digital DDH 381 values between the genome of $S$. aureus isolates and the closest relative genomes were 90.7100\% (using GBDP distance formula d0), 77.1-97.0\% (using GBDP distance formula d4), and 91.1-99.9\%(using GBDP distance formula d6). SA H27 and SA H32 genomes exhibit the nearest identities of $99.9 \%, 99.8 \%$, and $100 \%$ using the formula $\mathrm{d} 0, \mathrm{~d} 4$, and $\mathrm{d} 6$ respectively and displayed $\mathrm{G}+\mathrm{C}$ difference of $0.01 \%$. However, the SA G6 genome showed less identity to all the comparative genomes based on $\mathrm{dDDH}$. The high $\mathrm{G}+\mathrm{C}$ constituent difference $(0.1 \%)$ was observed in the case of isolated strains of SA G8 and SA H32 genomes. 
388 A whole-genome circular comparative map of four $S$. aureus genomes and their close reference 389 genomes was generated against $S$. aureus subsp. aureus HO 50960412 (HE681097.1) genome

390

391

392

393

394

395

396

397

398

399

400

401

402

403

404

405

406

407

408

409

410

411

412

413

414

415

416

417 using CGView server based on BLAST sequence similarities. Each genome was indicated by a different color, and the darker areas in the circular genome showed a 100\% sequence similarity with the reference genome, while the lighter (gray) areas showed a 70\% sequence similarity (Fig. 4). The map revealed less gap between the SA H27 (CP032161) and SA H32 (RAHP00000000) genomes showing high proximity between them when compared to other genomes. SA G6 (RAHA00000000) genome has many gaps with white color than the other genomes showing a distant relationship.

The SEED subsystem categories identified by RAST revealed that the genomes of all the isolates possessed "amino acids and derivatives" was the largest subsystem, followed by "carbohydrates", "protein metabolism", and "cofactors, vitamins, prosthetic groups, pigments" (Fig. 5A). The "carbohydrate", and "protein metabolism" subsystems were found largest in SA H27 (12.76\%) and SA H32 (10.47\%) genomes, respectively. The subsystem belongs to "phages, prophages, pathogenicity island" (2.5\%) was identified as highest in the SA G8 genome. Amongst the genomes, the SA G6 genome has the largest subsystem of "amino acids and derivatives" (15.9\%), and "virulence, disease, and defense" (4.43\%). In the "virulence, disease, and defense" subsystem of SA G6, 93 genes associated with adhesion, bacitracin stress response, colicin v, and bacteriocin production cluster, copper homeostasis, bile hydrolysis, cobalt-zinccadmium resistances, multidrug resistances, 2-protein, mercuric reductase, mercury resistance operon, streptothricin resistance, teicoplanin-resistances, aminoglycoside adenylyltransferases, fluoroquinolone resistances, arsenic resistance, fosfomycin resistance, beta-lactamase, cadmium resistance, multidrug resistance efflux pumps, and invasion and intracellular resistances. In the comparative eggNOG function study of $S$. aureus genomes, "amino acid transport and metabolism" was observed as for the majority of COGs, followed by those COGs related to "translation, ribosomal structure, and biogenesis", "transcription", and "cell wall/membrane/envelope". The eggNOG analysis results revealed that the SA G6 genome has the highest number of COGs associated with defense mechanisms (Fig. 5B). In the core genomes, $9.46 \%, 7.21 \%$, and $6.9 \%$ of COGs had functions associated to "amino acid transport and metabolism (E)", "translation, ribosomal structure, and biogenesis (J)", and "transcription 
418 (K)", respectively. Amongst the functional prediction of genomes, most COGs were associated 419 with "function unknown (S)".

420

421

422

423

424

425

426

427

428

429

430

431

432

433

434

435

436

437

438

439

440

441

442

443

444

445

446

447

448

\section{Pan-genome, core-genome, and singletons analysis}

The orthologous groups are categories into three groups based on the pan-genome distribution such as core (present in all genomes of $S$. aureus strains), dispensable (present in at least two strains, but not all), and singleton genes (present no orthologs in any other genomes). The comparison of four study $S$. aureus genomes generated a pan-genome size of 3265 genes, of which 2304 (70.6\%) genes were core genome, 462 (14.2\%) genes were dispensable, and 499 $(15.3 \%)$ genes were singletons. The Rcp value for the genomes of $S$. aureus isolates was calculated and the ratio Rcp was 0.70 and it is indicated that the genomes of $S$. aureus isolates were high inter-species diversity. A total of 499 singleton genes were calculated across the genomes of four $S$. aureus isolates, of which SA G6 genome acquired the highest number of singleton genes (220) that constitute the genes encode for proteins viz. aminoglycoside 3phosphotransferase, aminoglycoside 6-phosphotransferase, aminoglycoside N(6)acetyltransferase, streptothricin acetyltransferase, antiseptic resistance protein, cadmium resistance proteins, cadmium efflux system accessory protein, cadmium-transporting ATPase, ferric siderophore transport system, mercuric ion reductase, anti-adhesin, Tn552 transposase, pathogenicity islands (SaPI and SaPIn2), prophage-like elements, mobile elements, phage associated hypothetical proteins, hypothetical proteins, etc. The identified singleton genes of SA G6 genome were present within the genomic island (GI). This GI region is located between 2804353-2873411 base pair sequence region of the genomic sequence. While the SA H27 genome has the least singleton genes (6) constituting the genes encode for hypothetical proteins and phage proteins. The difference in the genomic constituents between the genome of SA H27 and SA H32 isolates revealed that SA H32 acquired the genes encoding for 23S rRNA (adenine(2058)-N(6))-dimethyltransferase, replication and maintenance protein, hypothetical proteins, phage-like elements, and mobile element protein. The genes shared by four study genomes and their respective singletons is represented in Fig. 6A. When the three reference $S$. aureus genomes were included in the pan-genome analysis, the core/pan-genome ratio drop down by $18.97 \%$ with inflation of pan-genome to 3415 genes and deflation of core-genome to 1762 genes. The core-genome and singleton genes formed by seven genomes of $S$. aureus strains is represented in flower-plot (Fig. 6B). When the three reference genomes of $S$. aureus strains 
449 were included in the pan-genome analysis, SA G6 isolate occupied the highest number of 450 singleton genes (104) while SA H27 isolate has the lowest singleton gene (2) (Fig. 6B). In the 451 pan-genome development analysis of seven $S$. aureus strains, $\alpha$ value (the power-law co-

452 efficient) was estimated as 0.141 which corresponds to the growing and open pan-genome model

453 (Fig. S2). The pan vs. core development plot appeared the progression of the pan and core454 genomes as additional genomes are added for analysis, and showing that the sharp decline of the 455 core-genome size with the introduction of S. aureus subsp. aureus ST228 (HE579073) (Fig. 6C). 456 In the plot of core-genome development, the core-genome size approach $(\Omega)$ value revealed that 457 the core-genome size of seven $S$. aureus would be declined to 1404.8 (Fig. S3). The singleton 458 development analysis suggested that the pan-genome size will continue to expand at the rate of 45935.9 genes per novel, representative genome (Fig. S4). The shape of the pan-genome vs. core460 genome curve showed fluctuation in their gene numbers when different order of the genomes 461 was set, even so, pan-genome and core genome developmental plots result remained unaffected 462 by the genomes order.

\section{Comparative phylogenetic tree analysis}

464 The phylogenomic analysis of $S$. aureus isolates provide the tree into three major clades (Fig.

465 7A). The clade A consists of 5 strains that belonged to CC22 and showing that Hungarian 466 isolates, SA H27 and SA H32 have the highest proximity. Germany isolates, SA G6 and SA G8 467 isolate and other strains belonged to CC5 were clustered in the clade C, showing that SA G6 468 isolate has closely relatedness to S. aureus subsp. aureus ST228 (HE579071.1) and the SA G8 469 isolate has a higher relatedness to S. aureus subsp. aureus JH9 (CP000703.1) than SA G6 isolate. 470 The phylogenetic relationship inferred from core-genome SNPs holds a similar agreement with 471 the whole genome-based phylogenetic analysis, and these methods could be useful in 472 distinguishing the genomes even in the strain level and phylogenetic trees are illustrated in Fig. 4737 7.

474 Discussion

475 S. aureus is a significant causative agent of both hospital and community-associated infections 476 (Chambers and DeLeo, 2009). The study of such pathogen at a molecular level through genome 477 comparative analysis improve the ideas of pathogenesis and evolution. Further, such a study 478 provides advantages in diagnosis, treatments, and infection controls (Kwong et al., 2015). In the 
479 present study, we used whole-genome sequencing (WGS) and in-silico analysis to determine the 480 comparative ARGs, VRGs, $\mathrm{pH}$ tolerance associated genes, and evolutionary relationship of four 481 S. aureus isolated from different sites of human infection such as skin, nostril, trachea, and 482 others.

483 The molecular epidemiology study of MRSA helps to find the risk factors associated with 484 MRSA infections and able to differentiate the several MRSA strains (Mistry et al., 2016). The genome-based molecular epidemiology studies found that Germany isolates exhibit SCCmec type I with ST228, and SCCmec type II with ST225 while Hungarian isolates hold SCCmec type IVa with ST22. Also, agr type II and I were owned by Germany and Hungarian isolates, respectively (Table 1). According to previous studies suggested that MRSA strains with SCCmec types I or II or III are dominant among the HA-MRSA, while SCCmec types IV or V are the characteristic of CA-MRSA (Monecke et al., 2011; Chua et al., 2014). The STs of

491

492

493

494

495

496

497

498

499

500

501

502

503

504

505

506

507

508

509

Germany isolates belonged to CC5 which is typical of HA-MRSA, while Hungarian isolates suggest its relationship CA-MRSA. In hospitals, the multidrug-resistance SCCmec type III was replaced by the multidrug-susceptible SCCmec type IV (ST22) strains slowly (D'Souza et al., 2010). The Hungarian isolates were found positive to Panton-Valentine Leukocidin (PVL) toxin, which is commonly used as a marker of CA-MRSA (Shukla et al., 2012; Singh et al., 2015) besides this toxin has shown to play a role necrosis, accelerating apoptosis and polynuclear- and mononuclear cells lysis, thereby contributing morbidity and mortality (Barrera-Rivas et al., 2017; Lina et al., 1999).

Staphylococcal $\beta$-lactamase encoded by blaZ gene is carried by the transposon $\operatorname{Tn} 552$ or $\operatorname{Tn} 552-$ like elements located on a large plasmid and can be non-inducible or inducible with antibiotics (Maddux, 1991). It was noticed that blaZ gene was absent in SA G8 isolate, probably due to the curing of blaZ positive plasmid (Pugazhendhi et al., 2020). Erythromycin resistance gene (ermA) was detected in the chromosome of SA G6, and SA G8 isolates, however, emrC gene was found in the plasmid of SA H32 (Fig. 1B). It was suggested that these genes may not be involved in the loss of specific ARGs for environmental adaptation, but it is expected to be essential for these isolates (Lim et al., 2015).

MRSA is responsible for causing biofilm infections that are more difficult to treat and need more intensive care as compared to Staphylococcus epidermidis biofilm (Reffuveille et al., 2017). The principal component of biofilm formation is PIA which consists of different intracellular

Peer) reviewing PDF | (2020:05:49105:2:0:NEW 17 Sep 2020) 
510 adhesion (ica) genes (Cramton et al., 1999) and play a crucial role in the initial stage of bacterial

511 cell adherence to surfaces and intercellular adhesion for the cells to aggregate (Farran et al.

512 2013). These genes were detected in all isolates however, the biofilm production ability varies

513 from weak to strong were observed in our previous study (Naorem et al., 2020). Our previous

514 study identified that SA G6 isolate obtained from skin infection showed a weak biofilm-forming 515 ability (Naorem et al., 2020). The low biofilm formation in SA G6 might be degraded the biofilm 516 by DNase enzyme found in skin cells (Eckhart et al., 2007). The previous study revealed that the 517 presence of the ica genes did not always correlate with biofilm (Møretrø et al., 2003; Nasr et al., 518 2012). Some authors reported that despite the presence of ica operon, some staphylococcal 519 isolates produce weak biofilm production due to the inactivation of $i c a A$ by insertion of IS256

520 (Cho et al., 2002; Kiem et al., 2004). Further reported that the insertion of IS256 inactivates $521 m u t S$ and contributes to vancomycin resistance development in vancomycin-intermediate $S$. 522 aureus strains (Kleinert et al., 2016). Also, the upregulation of icaA and icaD genes during acidic 523 stress promotes biofilm formation which in-turn plays a role to resist it from acidic and alkaline 524 environments and establishes the niche adaptation in Staphylococcus strains (Lindsay et al., 525 2006). In addition to ica locus, the presence of $c l f A, c l f B$, and epbs genes initiates the biofilm 526 formation (Ghasemian et al., 2015), however in the present study, the SA H27 isolate carried $527 c l f A$, and epbs genes and showed strong biofilm formation in our previous study (Naorem et al., 528 2020) compared to other isolates while SA G8 and SA H32 isolates carried $c l f A$, clfB, and epbs 529 genes though their biofilm formation was relatively low, suggesting that presence or absence of 530 such genes have no significant in biofilm formation. A recent study reported that $s d r C$ mutant 531 exhibited significantly inhibited biofilm formation (Chen et al., 2019) and the expression of 532 the ica operon and $s d r C$ are highly responsive to biofilm formation (Shin et al., 2013). Our study 533 revealed the sequence variation in $s d r C$ in Hungarian isolates, this variation might influence the 534 biofilm formation. The global regulatory gene, agr repression has been associated with biofilm 535 formation and its induction through AIP results in seeding dispersal in mature biofilm (Boles and 536 Horswill, 2008). CA-MRSA strains showed higher activity of $a g r$, which controls and enhance 537 the virulence (Aires-De-Sousa, 2017). It was reported that S. aureus strains belonged to agr I 538 group exhibited a strong biofilm-forming ability than the strains belonged to agr IV group 539 (Zhang et al., 2018) and a similar result was observed in one of our isolate SA H27. In addition 540 to this extracellular adherence protein (encoded by eap gene), and beta toxin (encoded by $h l b$ 
541 gene) play a role in biofilm maturation (Huseby et al., 2010; Sugimoto et al., 2013). In our

542 finding showed that eap gene was present only in the SA H27 isolate and this gene might be

543 attributed to high biofilm formation. Since biofilm formation involves many factors/genes that

544 take part in PIA dependent or independent biofilm, biofilm formation by regulator genes and

545 eDNA (Archer et al., 2011). Also, the presence of such genes in S. aureus may not provide much

546 impact on biofilm formation profiling. There was a difference in the prevalence of biofilm-

547 associated genes between the isolated strains and suggests that the presence of genes encoding

548 biofilm formation is not an absolute determinant of biofilm formation ability observed in our

549 previous study (Naorem et al., 2020). Thus, our future studies will focus on the expression

550 profiling of such relevant genes which may be necessary to determine the key genes involved in

551 biofilm formation.

552 The high survival rates were observed in both acidic and alkaline $\mathrm{pH}$ conditions in all isolates

553 was evaluated by the genomic study, it is elucidated that all the isolates possessed the arginine

554 deiminase and urease operon that aids in the generation of ammonia due to the hydrolysis of L-

555 arginine and urea by arginine deiminase and urease. The released ammonia and urea counteract

556 the acidic environment (Cotter and Hill, 2003; Valenzuela et al., 2003). Further, the proton efflux

557 pump $\left(\mathrm{F}_{0} \mathrm{~F}_{1}\right.$ ATPase $)$ plays a role to extrude $\mathrm{H}^{+}$out of the cells and maintains the $\mathrm{pH}$

558 homeostasis (Foster, 2004; Maurer et al., 2005). However, in the case of alkaline tolerance, it

559 was reported that the $S$. aureus genome encodes a unique Ktr-like system where the cytoplasmic

560 gating protein $\mathrm{KtrC}$ regulates the uptake of $\mathrm{K}^{+}$that is essential for maintaining cytoplasmic $\mathrm{pH}$

561 and supporting $\mathrm{H}^{+}$efflux under alkaline conditions (Gries et al., 2016).

562 The ability of $S$. aureus as a pathogen can be accredited to its arsenal of virulence factors among

563 which secreted pore-forming toxins (PFTs), exfoliative toxins (ETs), ESAT-6-like proteins,

564 exoenzymes, and superantigens (SAgs) play a significant role in the pathogenesis of invading

565 infections in healthy individuals (Otto et al., 2014, Bartiett et al., 2010). The presence of $h l b$ gene

566 in the isolates contributes to the phagosomal escape of $S$. aureus and influences biofilm

567 development (Huseby et al., 2010, Periasamy et al., 2012). The PVL toxin was identified in the

568 prophages of Hungarian isolates and expressing Sa2 integrase. These isolates have cytolytic

569 activity against blood cells and leukocytes, contributing to the $S$. aureus pathogenicity

570 (Vandenesch et al., 2003). Staphylococcal enterotoxins (SEs) or staphylococcal superantigens

571 proteins (SAgs) are well-known for causing food poisoning, localized epidermal infections 
572 (bullous impetigo), and generalized diseases (Staphylococcal scalded skin syndrome) (Grumann

573 et al., 2014, Argudín et al., 2010). SEs encoding genes are located on mobile elements including

574 bacteriophages, pathogenicity islands (SaPI), or plasmids. In this study, SEs encoding genes such

575 as sea, seg, sei, yent1, yent2, selk, selm, seln, and selo were identified. Hungarian isolates, SA

$576 \mathrm{H} 27$, and SA H32 acquired seg and sei genes, however sei gene was absent in Germany isolates,

577 SA G6, and SA G8. These seg and sei genes belong to egc (enterotoxin gene cluster), involve in

578 staphylococcal food poisoning TSS, and SSF (Jarraud et al., 2001, Chen et al., 2004) and egc

579 was distributed widely in clinical isolates and playing a role in pathogenesis (Jarraud et al.,

580 2001). Exfoliative toxins (ETs) are known as epidermolytic toxins that induce skin shedding and

581 blister formation (Melish and Glasgow, 1971). In this study, eta gene encoded for ETA toxin was

582 found in all the isolates and responsible for causing human skin damage, and most prevalent in

583 Europe (Ladhani, 2001). Capsular polysaccharide synthesis genes are almost all detected in

584 clinical isolates $S$. aureus showing significant virulence by targeting the antibodies that protect

585 against Staphylococcal infections (Su et al., 1997). Type VII secretion system (T7SS) was

586 present in Germany isolates (Fig. 3A) and promoting them to persist in their hosts (Tchoupa et

587 al., 2019). The esxA and esxB gene show a significant role in the distribution and colonization of

588 S. aureus, and activation of the cell-mediated immune responses, boost the pathogenesis (Burts

589 et al., 2005). Also, esaD gene found only in Germany isolates suggesting that this gene can

590 inhibit the growth of other closely related $S$. aureus strains and playing a role in an intra-species

591 competition (Cao et al., 2016). The family of beta-hemolysin converting phage encodes proteins

592 such as SCIN (staphylococcal complement inhibitor) and CHIPS (chemotaxis inhibiting protein

593 of staphylococcus) involved in host-pathogen interaction and contribute to evading human innate

594 immune response (Wamel et al., 2006), these proteins were identified in intact prophages of SA

595 G8 and SA G27 genomes but CHIPs was absent in the prophages of SA H32 genome. Therefore,

596 prophages were the reservoir of virulence and resistance factors that play a role in the evolution

597 of virulence strains and causing a major threat to human and animal health (Barrera-Rivas et al.,

598 2017). The presence of ARGs and VFGs in the prophage regions of SA G6 genome differentiates

599 it from the other $S$. aureus isolates and may determine its greater pathogenic potential by

600 modifying its antigenicity (Barrera-Rivas et al., 2017). Also, plasmid p1G6 carried qacA gene,

601 which is known to decrease chlorhexidine (antiseptic) susceptibility and giving an event of

602 MGEs transfer evidence of qacA across the $S$. aureus strains (LaBreck et al., 2018). The harbor

Peer] reviewing PDF | (2020:05:49105:2:0:NEW 17 Sep 2020) 
603 of MGEs (mosaic features of prophages and plasmids) contributes to the tremendous distribution 604 of ARGs and VFGs among the S. aureus isolates (McCarthy and Lindsay, 2012; McCarthy et al., 605 2014). This MGEs transfer event could be useful for the survival of S. aureus in different 606 ecological niches (Lindsay, 2010).

607 The pangenome described here is composed of 3415 genes, of these, 1762 genes are shared 608 among $S$. aureus isolates (Fig. 6B). Functional annotation of the core-genome revelated that they 609 are mostly associated transcription and translation, and different metabolism categories, such 610 similar result was reported previously (Bosi et al., 2016; Sharma et al., 2018). The core-genome 611 and accessory genome functional characterizations revealed that $S$. aureus isolates required 612 amino acids than carbohydrates as the energy source and suggests that these isolates adapted to 613 grow in a protein-rich medium than carbohydrates (Fig. 5A and 5B). It was suggested that the 614 survival of $S$. aureus can be maintained by the catabolism of amino acids (Halsey et al., 2017). 615 The core-genome has $51.6 \%$ of genes and validated that $S$. aureus is a clonal species (Feil et al., 616 2003; Bosi et al., 2016). The mutation event that occurred in the core-genome of closely related 617 S. aureus provides important roles in virulence and persistence of $S$. aureus strains (Kennedy et 618 al., 2008). So, an in-depth analysis of strain-specific genetic variation is required for further 619 understanding of the pathogenicity. The inflation of pan-genome and deflation of core-genome 620 was seen after the introduction of reference genomes and its regression analysis revealed that the 621 pan-genome is open, suggesting that the gene repertoire of this species is theoretically limitless. 622 A similar finding was observed in the DNA microarray experiment of thirty-six S. aureus 623 isolates (Fitzgerald et al., 2001). The drastic decline of the core/pan- genome ratio after the 624 introduction HE579071.1 (S. aureus subsp. aureus ST228) and SA G6 suggested that these two 625 strains have distinct genomic contents (Fig. 6C). The genomic content variation between the 626 genomes is due to the acquisition of certain genes that encode for virulence and resistance 627 factors, pathogenicity islands, prophage-like elements, plasmids, mobile element proteins, and 628 hypothetical proteins in the GIs. These GIs are mobilized across organisms via HGT events 629 (Schmidt and Hensel, 2004). This finding was supported by gaps that appeared in the genome 630 ring of SA G6 genome and suggesting that this isolate showed a distant relationship to others 631 (Fig.4). The gaps that appeared in the map are due to the GC\% content difference in the 632 comparative genomes, and it results from the event of MGEs transfer via HGT and the GC 633 skewed regions indicated the regions where HGT occurred (Hayek, 2013). 
634 We specifically analyzed the presence of ARGs and VFGs in the core genomes and pangenomes.

635 Some genes involved in multidrug resistance or drug efflux such ygaD, arlR, arlS, and mepA are 636 components of the core-genome (Fig. 2). The large repertoire of genes (29\%) in the accessory 637 genome gives advantages in adaptation and that can contribute to pathogenicity or niche 638 specificity of strains (Medini et al., 2005). The analysis of pangenome is essential to understand 639 the event of MGEs transfer and S. aureus evolution (Ozer, 2018). The interpretation from the 640 dispersible and singleton genes content analysis of $S$. aureus genomes allows us to understand 641 the genetic variation among the CC5 and CC22. Juhas et al. reported that most dispensable and 642 singleton genes were acquired through HGT and operate an important role in drug resistance or 643 virulence (Juhas et al., 2012). A high portion of unique genes or singletons in S. aureus genomes 644 were related to MGEs, which could drive the gaining of novel functional elements especially 645 drug resistance and virulence. These singletons are the main drivers of the phenotypic variation 646 within $S$. aureus strains and the evolution of S. aureus (Carvalho et al., 2019).

647 The phylogenetic trees based on whole-genome and core-genome SNP methods support each 648 other and revealed that these methods were able to distinguish between strains at a higher 649 resolution in terms of the geographic origin of strains and phylogenetic trees are illustrated in 650 Fig. 7. The phylogenomic analysis revealed that the strains with ST225 (Germany), ST228 651 (Germany, Switzerland), ST105 (USA), and ST5 (Japan) were clustered in the same CC5 clade 652 (Cluster C), and a different clade (Cluster A) was noticed among the UK origin ST22 (CC22) 653 and diverged from Germany origin strains (Fig. 7A), this finding was in good agreement with the 654 previously published article (Aanensen et al., 2016). The CC5 (ST225) and CC22 (ST22) were 655 found to be the most dominant clones circulating in Europe (Grundmann et al., 2014). The 656 comparative genome analysis revealed that Germany and Hungarian isolates are genetically 657 diverse and showing variation among them due to the gain or loss of MGEs such as SCCmec, 658 plasmid, phage elements, or the insertion of transposase. The event of MGEs transfer was 659 observed in ST5, ST225, and ST228 (Fig. 7A), and similar results were also reported previously 660 (Nübel et al., 2008; Nübel et al., 2010; Vogel et al., 2012). The SNPs located in the core-genome 661 define as the element present in S. aureus strains, these SNPs based phylogenetic tree was 662 constructed to avoid the HGT of MGEs misuse phylogenetic interpretation, as well as this tree, 663 resolved the subdivision within cluster C of Fig.7A indicating that SA G6 isolates and S. aureus 664 subsp. aureus ST228 exhibits the closest strains (Fig. 7B). These strains shared the genetic 
665 background (ST228/SCCmec-I) and revealing 99.8\% OrthoANIu similarity value in their 666 genomes, likewise, Hungarian isolates (SA H27 and SA H32) in clade A (Fig. 7B) shared 667 molecular epidemiological background in terms of SCCmec-IVa, and ST-22 and showing 99.8\% 668 OrthoANIu value. However, SA G8 isolate and S. aureus subsp. aureus ST228 belongs to ST225 669 and ST105, respectively were clustered together (Fig. 7B). The strains with the same genetic 670 background were clustered together in both phylogenetic trees which suggest that these strains 671 are highly alike, however comparative genome analysis exposed that the acquisition of phage 672 elements and plasmids through the events of MGEs transfer contribute to differences in their 673 phenotypic characters. Such events provide an impact on the fitness or pathogenicity or 674 epidemicity of the strains.

\section{Conclusions}

676 Using WGS, we characterized the four clinical MRSA isolates that infect the skin, nostrils, 677 trachea, and other sites. The data generated from the WGS confirmed the diversity of MRSA 678 among the same CC5 and CC22. It is clearly stated that the biofilm-forming ability of MRSA 679 was not correlated with the presence of biofilm-forming encoding genes, also the genetic 680 constituents have no information regarding the infection sites. So, expression profiling of 681 biofilm-related genes is required to define the key genes involved in biofilm formation. The 682 comparative genome study allowed the segregation of isolates of geographical origin, and 683 differentiation of clinical isolates from the commensal isolates. An interesting finding is the 684 addition of SA G6 genome responsible for open pan-genome and diversity among genomes. The 685 openness of pan-genomes of $S$. aureus isolates relies on the acquisition of MGEs. The evidence 686 of MGEs transfer event especially in SA G6 is supported by the drastic drop of the core/pan687 genome ratio curve, and gaps and GC skewed regions in comparative genome map. The presence 688 of ant(6)-Ia, aph(3')-III) and sat-4 in the GI region of SA G6 are likely acquired and these genes 689 may provide fitness and a selective advantage during host-adaptation and colonization.

690 Phylogenetic analysis suggests that SA G6 and S. aureus subsp. aureus ST228 strains are distinct 691 from its group. The acquisition of plasmid and prophage functional modules such as ARGs and 692 VFGs in S. aureus isolates contributes a major role in the rapid evolution of pathogenic S. aureus 693 lineages and that confer specific advantages in a defined host under environmental conditions. 694 Through this comparative genome analysis would improve the knowledge about the pathogenic 
695 S. aureus strain's characterization, adaptation, and dynamic evolutionary process in the 696 transmission of infections globally.

\section{Acknowledgments}

698 We acknowledged Dr. Schneider Gyorgy, Department of Medical Microbiology and 699 Immunology, Medical School, University of Pecs for providing the strains. Further, we 700 acknowledged Peter Urban for technical laboratory assistance and Gunajit Goswami for English 701 language review. The authors acknowledge the anonymous reviewers for their valuable 702 suggestions that helped improve the quality of the manuscript.

703

704

705

706

707

708

709

710

711

712

713

714

715

716

717

718

719

720

\section{References}

Aanensen DM., Feil EJ., Holden MTG., Dordel J., Yeats CA., Fedosejev A., Goater R., CastilloRamírez S., Corander J., Colijn C., Chlebowicz MA., Schouls L., Heck M., Pluister G., Ruimy R., Kahlmeter G., Åhman J., Matuschek E., Friedrich AW., Parkhill J., Bentley SD., Spratt BG., Grundmann H. 2016. Whole-Genome Sequencing for Routine Pathogen Surveillance in Public Health: a Population Snapshot of Invasive Staphylococcus aureus in Europe. mBio 7. DOI: 10.1128/mbio.00444-16.

Aires-De-Sousa M. 2017. Methicillin-resistant Staphylococcus aureus among animals: current overview. Clinical Microbiology and Infection 23:373-380. DOI: 10.1016/j.cmi.2016.11.002.

Alcock BP., Raphenya AR., Lau TTY., Tsang KK., Bouchard M., Edalatmand A., Huynh W., Nguyen A-LV., Cheng AA., Liu S., Min SY., Miroshnichenko A., Tran H-K., Werfalli RE., Nasir JA., Oloni M., Speicher DJ., Florescu A., Singh B., Faltyn M., HernandezKoutoucheva A., Sharma AN., Bordeleau E., Pawlowski AC., Zubyk HL., Dooley D., Griffiths E., Maguire F., Winsor GL., Beiko RG., Brinkman FSL., Hsiao WWL., Domselaar GV., Mcarthur AG. 2019. CARD 2020: antibiotic resistome surveillance with the comprehensive antibiotic resistance database. Nucleic Acids Research. DOI: 10.1093/nar/gkz935. 
721

722

723

Archer NK., Mazaitis MJ., Costerton JW., Leid JG., Powers ME., Shirtliff ME. 2011. Staphylococcus aureusbiofilms. Virulence 2:445-459. DOI: 10.4161/viru.2.5.17724.

Argudín MÁ., Mendoza MC., Rodicio MR. 2010. Food Poisoning and Staphylococcus aureus Enterotoxins. Toxins 2:1751-1773. DOI: 10.3390/toxins2071751.

Arndt D., Grant JR., Marcu A., Sajed T., Pon A., Liang Y., Wishart DS. 2016. PHASTER: a better, faster version of the PHAST phage search tool. Nucleic Acids Research 44. DOI: 10.1093/nar/gkw387.

Aziz RK., Bartels D., Best AA., Dejongh M., Disz T., Edwards RA., Formsma K., Gerdes S., Glass EM., Kubal M., Meyer F., Olsen GJ., Olson R., Osterman AL., Overbeek RA., Mcneil LK., Paarmann D., Paczian T., Parrello B., Pusch GD., Reich C., Stevens R., Vassieva O., Vonstein V., Wilke A., Zagnitko O. 2008. The RAST Server: Rapid Annotations using Subsystems Technology. BMC Genomics 9:75. DOI: 10.1186/14712164-9-75.

Barrera-Rivas CI., Valle-Hurtado NA., González-Lugo GM., Baizabal-Aguirre VM., BravoPatiño A., Cajero-Juárez M., Valdez-Alarcón JJ. 2017. Bacteriophage Therapy: An Alternative for the Treatment of Staphylococcus aureus Infections in Animals and Animal Models. Frontiers in Staphylococcus aureus. DOI: 10.5772/65761.

Bartels MD., Petersen A., Worning P., Nielsen JB., Larner-Svensson H., Johansen HK., Andersen LP., Jarlov JO., Boye K., Larsen AR., Westh H. 2014. Comparing WholeGenome Sequencing with Sanger Sequencing for spa Typing of Methicillin-Resistant Staphylococcus aureus. Journal of Clinical Microbiology 52:4305-4308. DOI: 10.1128/jcm.01979-14.

Bartlett AH., Hulten KG. 2010. Staphylococcus aureus Pathogenesis. The Pediatric Infectious Disease Journal 29:860-861. DOI: 10.1097/inf.0b013e3181ef2477.

Bertelli C, Laird MR, Williams KP, Simon Fraser University Research Computing Group, Lau BY, Hoad G, Winsor GL, Brinkman FS. 2017. IslandViewer 4: expanded prediction of 
747

748

749

750

751

752

753

754

755

756

757

758

759

760

761

762

763

764

765

766

767

768

769

770

genomic islands for larger-scale datasets. Nucleic Acids Research 45:W30-W35. doi:10.1093/nar/gkx343.

Bikandi J., Millan RS., Rementeria A., Garaizar J. 2004. In silico analysis of complete bacterial genomes: PCR, AFLP-PCR and endonuclease restriction. Bioinformatics 20:798-799. DOI: 10.1093/bioinformatics/btg491.

Blin K., Shaw S., Steinke K., Villebro R., Ziemert N., Lee SY., Medema MH., Weber T. 2019. antiSMASH 5.0: updates to the secondary metabolite genome mining pipeline. Nucleic Acids Research 47. DOI: 10.1093/nar/gkz310.

Blom J., Kreis J., Spänig S., Juhre T., Bertelli C., Ernst C., Goesmann A. 2016. EDGAR 2.0: an enhanced software platform for comparative gene content analyses. Nucleic Acids Research 44. DOI: 10.1093/nar/gkw255.

Boles BR., Horswill AR. 2008. agr-Mediated Dispersal of Staphylococcus aureus Biofilms. PLoS Pathogens 4. DOI: 10.1371/journal.ppat.1000052.

Bosi E., Donati B., Galardini M., Brunetti S., Sagot M-F., Lió P., Crescenzi P., Fani R., Fondi M. 2015. MeDuSa: a multi-draft based scaffolder. Bioinformatics 31:2443-2451. DOI: 10.1093/bioinformatics/btv171.

Bosi E., Monk JM., Aziz RK., Fondi M., Nizet V., Palsson BØ. 2016. Comparative genomescale modelling of Staphylococcus aureus strains identifies strain-specific metabolic capabilities linked to pathogenicity. Proceedings of the National Academy of Sciences 113. DOI: $10.1073 /$ pnas. 1523199113.

Burts ML., Williams WA., Debord K., Missiakas DM. 2005. EsxA and EsxB are secreted by an ESAT-6-like system that is required for the pathogenesis of Staphylococcus aureus infections. Proceedings of the National Academy of Sciences 102:1169-1174. DOI: 10.1073/pnas.0405620102. 
771 Cao Z., Casabona MG., Kneuper H., Chalmers JD., Palmer T. 2016. The type VII secretion

772

773

774

775

776

777

778

779

780

781

782

783

784

785

786

787

788

789

790

791

792

793

794

795

system of Staphylococcus aureus secretes a nuclease toxin that targets competitor bacteria. Nature Microbiology 2. DOI: 10.1038/nmicrobiol.2016.183.

Carattoli A., Zankari E., García-Fernández A., Larsen MV., Lund O., Villa L., Aarestrup FM., Hasman H. 2014. In SilicoDetection and Typing of Plasmids using PlasmidFinder and Plasmid Multilocus Sequence Typing. Antimicrobial Agents and Chemotherapy 58:38953903. DOI: 10.1128/aac.02412-14.

Carvalho SPD., Almeida JBD., Freitas LMD., Guimarães AMS., Nascimento NCD., Santos APD., Campos GB., Messick JB., Timenetsky J., Marques LM. 2019. Genomic profile of Brazilian methicillin-resistant Staphylococcus aureus resembles clones dispersed worldwide. Journal of Medical Microbiology 68:693-702. DOI: 10.1099/jmm.0.000956.

Chaieb K., Mahdouani K., Bakhrouf A. 2005. Detection of icaA and icaD loci by polymerase chain reaction and biofilm formation by Staphylococcus epidermidis isolated from dialysate and needles in a dialysis unit. Journal of Hospital Infection 61:225-230. DOI: 10.1016/j.jhin.2005.05.014.

Chambers HF., Deleo FR. 2009. Waves of resistance: Staphylococcus aureus in the antibiotic era. Nature Reviews Microbiology 7:629-641. DOI: 10.1038/nrmicro2200.

Chan CX., Beiko RG., Ragan MA. 2011. Lateral Transfer of Genes and Gene Fragments in Staphylococcus Extends beyond Mobile Elements. Journal of Bacteriology 193:39643977. DOI: 10.1128/jb.01524-10.

Chen Q., Xie S., Lou X., Cheng S., Liu X., Zheng W., Zheng Z., Wang H. 2019. Biofilm formation and prevalence of adhesion genes among Staphylococcus aureus isolates from different food sources. Microbiology Open 9. DOI: 10.1002/mbo3.946.

Chen T-R., Chiou C-S., Tsen H-Y. 2004. Use of novel PCR primers specific to the genes of staphylococcal enterotoxin G, H, I for the survey of Staphylococcus aureus strains isolated 
796

797

798

799

800

801

802

803

804

805

806

807

808

809

810

811

812

813

814

815

816

817

818

819

820

from food-poisoning cases and food samples in Taiwan. International Journal of Food Microbiology 92:189-197. DOI: 10.1016/j.ijfoodmicro.2003.10.002.

Cheng J., Wang Y., Cao Y., Yan W., Niu X., Zhou L., Chen J., Sun Y., Li C., Zhang X., Wu Y. 2016. The Distribution of 18 Enterotoxin and Enterotoxin-Like Genes in Staphylococcus aureus Strains from Different Sources in East China. Foodborne Pathogens and Disease 13:171-176. DOI: 10.1089/fpd.2015.1963.

Cho S-H., Naber K., Hacker J., Ziebuhr W. 2002. Detection of the icaADBC gene cluster and biofilm formation in Staphylococcus epidermidis isolates from catheter-related urinary tract infections. International Journal of Antimicrobial Agents 19:570-575. DOI: 10.1016/s0924-8579(02)00101-2.

Chua KY., Howden BP., Jiang J-H., Stinear T., Peleg AY. 2014. Population genetics and the evolution of virulence in Staphylococcus aureus. Infection, Genetics and Evolution 21:554-562. DOI: 10.1016/j.meegid.2013.04.026.

Cotter PD., Hill C. 2003. Surviving the Acid Test: Responses of Gram-Positive Bacteria to Low pH. Microbiology and Molecular Biology Reviews 67:429-453. DOI: 10.1128/mmbr.67.3.429-453.2003.

Cramton SE., Gerke C., Schnell NF., Nichols WW., Götz Friedrich. 1999. The Intercellular Adhesion (ica) Locus Is Present in Staphylococcus aureus and Is Required for Biofilm Formation. Infection and Immunity 67:5427-5433. DOI: 10.1128/iai.67.10.54275433.1999 .

Darling AE., Mau B., Perna NT. 2010. progressiveMauve: Multiple Genome Alignment with Gene Gain, Loss and Rearrangement. PLoS ONE 5. DOI: 10.1371/journal.pone.0011147.

Deleo FR., Diep BA., Otto M. 2009. Host Defense and Pathogenesis in Staphylococcus aureus Infections. Infectious Disease Clinics of North America 23:17-34. DOI: 10.1016/j.idc.2008.10.003. 
821 Du J., Chen C., Ding B., Tu J., Qin Z., Parsons C., Salgado C., Cai Q., Song Y., Bao Q., Zhang

822 L., Pan J., Wang L., Yu F. 2011. Molecular Characterization and Antimicrobial

823 Susceptibility of Nasal Staphylococcus aureus Isolates from a Chinese Medical College

824 Campus. PLoS ONE 6. DOI: 10.1371/journal.pone.0027328.

825

826

827

828

829

830

831

832

833

834

835

836

837

838

839

840

841

842

843

844 845

Eckhart L., Fischer H., Barken K., Tolker-Nielsen T., Tschachler E. 2007. DNase1L2 suppresses biofilm formation by Pseudomonas aeruginosa and Staphylococcus aureus. British Journal of Dermatology 156:1342-1345. DOI: 10.1111/j.1365-2133.2007.07886.x.

Farran CE., Sekar A., Balakrishnan A., Shanmugam S., Arumugam P., Gopalswamy J. 2013. Prevalence of biofilm-producing Staphylococcus epidermidis in the healthy skin of individuals in Tamil Nadu, India. Indian Journal of Medical Microbiology 31:19. DOI: 10.4103/0255-0857.108712.

Farris JS. 1972. Estimating Phylogenetic Trees from Distance Matrices. The American Naturalist 106:645-668. DOI: 10.1086/282802.

Feil EJ., Cooper JE., Grundmann H., Robinson DA., Enright MC., Berendt T., Peacock SJ., Smith JM., Murphy M., Spratt BG., Moore CE., Day NPJ. 2003. How Clonal Is Staphylococcus aureus? Journal of Bacteriology 185:3307-3316. DOI: 10.1128/jb.185.11.3307-3316.2003.

Fitzgerald JR., Sturdevant DE., Mackie SM., Gill SR., Musser JM. 2001. Evolutionary genomics of Staphylococcus aureus: Insights into the origin of methicillin-resistant strains and the toxic shock syndrome epidemic. Proceedings of the National Academy of Sciences 98:8821-8826. DOI: 10.1073/pnas.161098098.

Foster JW. 2004. Escherichia coli acid resistance: tales of an amateur acidophile. Nature Reviews Microbiology 2:898-907. DOI: 10.1038/nrmicro1021.

Galata V., Fehlmann T., Backes C., Keller A. 2018. PLSDB: a resource of complete bacterial plasmids. Nucleic Acids Research 47. DOI: 10.1093/nar/gky1050. 
846 Ghasemian, A., Najar Peerayeh, S., Bakhshi, B., \& Mirzaee, M. 2015. The Microbial Surface 847 Components Recognizing Adhesive Matrix Molecules (MSCRAMMs) Genes among 848 Clinical Isolates of Staphylococcus aureus from Hospitalized Children. Iranian journal of 849 pathology, 10(4), 258-264.

850 Ghatak S., Blom J., Das S., Sanjukta R., Puro K., Mawlong M., Shakuntala I., Sen A., Goesmann

851

852

853

854

855

856

857

858

859

860

861

862

863

864

865

866

867

868

869

870

A., Kumar A., Ngachan SV. 2016. Pan-genome analysis of Aeromonas hydrophila, Aeromonas veronii and Aeromonas caviae indicates phylogenomic diversity and greater pathogenic potential for Aeromonas hydrophila. Antonie van Leeuwenhoek 109:945-956. DOI: $10.1007 / \mathrm{s} 10482-016-0693-6$.

Götz F., Bannerman T., Schleifer K-H. 2006. The Genera Staphylococcus and Macrococcus. The Prokaryotes:5-75. DOI: 10.1007/0-387-30744-3_1.

Goudarzi, M., Mobarez, A. M., Najar-Peerayeh, S., \& Mirzaee, M. 2018. Prevalence of biofilm formation and vancomycin-resistant genes among Enterococcus faecium isolated from clinical and environmental specimens in Lorestan hospitals. Iranian journal of microbiology, 10(2), 74-81.

Gould I. 2005. The clinical significance of methicillin-resistant Staphylococcus aureus. Journal of Hospital Infection 61:277-282. DOI: 10.1016/j.jhin.2005.06.014.

Grant JR., Stothard P. 2008. The CGView Server: a comparative genomics tool for circular genomes. Nucleic Acids Research 36. DOI: 10.1093/nar/gkn179.

Gries CM., Sadykov MR., Bulock LL., Chaudhari SS., Thomas VC., Bose JL., Bayles KW. 2016. Potassium Uptake Modulates Staphylococcus aureus Metabolism. mSphere 1. DOI: 10.1128/msphere.00125-16.

Grumann D., Nübel U., Bröker BM. 2014. Staphylococcus aureus toxins - Their functions and genetics. Infection, Genetics and Evolution 21:583-592. DOI:

10.1016/j.meegid.2013.03.013.

Peer] reviewing PDF | (2020:05:49105:2:0:NEW 17 Sep 2020) 
871 Grundmann H., Schouls LM., Aanensen DM., Pluister GN., Tami A., Chlebowicz M., Glasner 872 C., Sabat AJ., Weist K., Heuer O., Friedrich AW., Collective On Behalf Of The Escmid 873 Study Group. 2014. The dynamic changes of dominant clones of Staphylococcus aureus 874 causing bloodstream infections in the European region: Results of a second structured 875 survey. Eurosurveillance 19. DOI: 10.2807/1560-7917.es2014.19.49.20987.

876

877

878

879

880

881

882

883

884 885

886

887

888

889

890

891

892

893

894

Gurevich A., Saveliev V., Vyahhi N., Tesler G. 2013. QUAST: quality assessment tool for genome assemblies. Bioinformatics 29:1072-1075. DOI: 10.1093/bioinformatics/btt086.

Halsey, C. R., Lei, S., Wax, J. K., Lehman, M. K., Nuxoll, A. S., Steinke, L., Sadykov, M., Powers, R., \& Fey, P. D. (2017). Amino Acid Catabolism in Staphylococcus aureus and the Function of Carbon Catabolite Repression. mBio, 8(1), e01434-16. https://doi.org/10.1128/mBio.01434-16

Hayek N. 2013. Lateral transfer and GC content of bacterial resistant genes. Frontiers in Microbiology 4. DOI: 10.3389/fmicb.2013.00041.

Huerta-Cepas J., Szklarczyk D., Heller D., Hernández-Plaza A., Forslund SK., Cook H., Mende DR., Letunic I., Rattei T., Jensen LJ., Von Mering C., Bork P. 2018. eggNOG 5.0: a hierarchical, functionally and phylogenetically annotated orthology resource based on 5090 organisms and 2502 viruses. Nucleic Acids Research 47. DOI: 10.1093/nar/gky1085.

Hughes AL., Friedman R. 2005. Nucleotide Substitution and Recombination at Orthologous Loci in Staphylococcus aureus. Journal of Bacteriology 187:2698-2704. DOI: 10.1128/jb.187.8.2698-2704.2005.

Huseby MJ., Kruse AC., Digre J., Kohler PL., Vocke JA., Mann EE., Bayles KW., Bohach GA., Schlievert PM., Ohlendorf DH., Earhart CA. 2010. Beta toxin catalyzes formation of nucleoprotein matrix in staphylococcal biofilms. Proceedings of the National Academy of Sciences 107:14407-14412. DOI: 10.1073/pnas.0911032107. 
895 Jansen W., Vanderbruggen J., Verhoef J., Fluit A. 2006. Bacterial resistance: A sensitive issue 896 Complexity of the challenge and containment strategy in Europe. Drug Resistance Updates 897 9:123-133. DOI: 10.1016/j.drup.2006.06.002.

898

899

900

901

902

903

904

905

906

907

908

909

910

911

912

913

914

915

916

917

918

919

Jarraud S., Peyrat MA., Lim A., Tristan A., Bes M., Mougel C., Etienne J., Vandenesch F., Bonneville M., Lina G. 2001. egc, A Highly Prevalent Operon of Enterotoxin Gene, Forms a Putative Nursery of Superantigens in Staphylococcus aureus. The Journal of Immunology 166:669-677. DOI: 10.4049/jimmunol.166.1.669.

Juhas M, Eberl L, Church GM. 2012. Essential genes as antimicrobial targets and cornerstones of synthetic biology. Trends in Biotechnology 30: 601-607.

Kaas RS., Leekitcharoenphon P., Aarestrup FM., Lund O. 2014. Solving the Problem of Comparing Whole Bacterial Genomes across Different Sequencing Platforms. PLoS ONE 9. DOI: 10.1371/journal.pone.0104984.

Kaya H., Hasman H., Larsen J., Stegger M., Johannesen TB., Allesøe RL., Lemvigh CK., Aarestrup FM., Lund O., Larsen AR. 2018. SCCmecFinder, a Web-Based Tool for Typing of Staphylococcal Cassette Chromosome mec in Staphylococcus aureus Using WholeGenome Sequence Data. mSphere 3. DOI: 10.1128/msphere.00612-17.

Kennedy AD., Otto M., Braughton KR., Whitney AR., Chen L., Mathema B., Mediavilla JR., Byrne KA., Parkins LD., Tenover FC., Kreiswirth BN., Musser JM., Deleo FR. 2008. Epidemic community-associated methicillin-resistant Staphylococcus aureus: Recent clonal expansion and diversification. Proceedings of the National Academy of Sciences 105:1327-1332. DOI: 10.1073/pnas.0710217105.

Kiem S., Oh WS., Peck KR., Lee NY., Lee J-Y., Song J-H., Hwang ES., Kim E-C., Cha CY., Choe K-W. 2004. Phase Variation of Biofilm Formation in Staphylococcus aureus by IS256Insertion and Its Impact on the Capacity Adhering to Polyurethane Surface. Journal of Korean Medical Science 19:779. DOI: 10.3346/jkms.2004.19.6.779. 
920 Kleinert F., Kallies R., Zweynert A., Bierbaum G. 2016. Draft Genome Sequences of Three 921 Northern German Epidemic Staphylococcus aureus (ST247) Strains Containing Multiple Copies of IS 256 : TABLE 1. Genome Announcements 4. DOI: 10.1128/genomea.00936-

924

925

926

927

928

929

930

931

932

933

934

935

936

937

938

939

940

941

942

943

944

945 16.

Köser CU., Ellington MJ., Cartwright EJP., Gillespie SH., Brown NM., Farrington M., Holden MTG., Dougan G., Bentley SD., Parkhill J., Peacock SJ. 2012. Routine Use of Microbial Whole Genome Sequencing in Diagnostic and Public Health Microbiology. PLoS Pathogens 8. DOI: 10.1371/journal.ppat.1002824.

Kumurya A. 2015. One step PCR for detection of Staphylococcus aureus specific sequence gene and mecA gene in Northwestern Nigerian hospitals. Antimicrobial Resistance and Infection Control 4. DOI: 10.1186/2047-2994-4-s1-p196.

Kwong J., Mccallum N., Sintchenko V., Howden B. 2015. Whole genome sequencing in clinical and public health microbiology. Pathology 47:199-210. DOI: 10.1097/pat.0000000000000235.

Labreck PT., Rice GK., Paskey AC., Elassal EM., Cer RZ., Law NN., Schlett CD., Bennett JW., Millar EV., Ellis MW., Hamilton T., Bishop-Lilly KA., Merrell DS. 2018. Conjugative Transfer of a Novel Staphylococcal Plasmid Encoding the Biocide Resistance Gene, qacA. Frontiers in Microbiology 9. DOI: 10.3389/fmicb.2018.02664.

Ladhani S. 2001. Recent developments in staphylococcal scalded skin syndrome. Clinical Microbiology and Infection 7:301-307. DOI: 10.1046/j.1198-743x.2001.00258.x.

Laing C., Buchanan C., Taboada EN., Zhang Y., Kropinski A., Villegas A., Thomas JE., Gannon VP. 2010. Pan-genome sequence analysis using Panseq: an online tool for the rapid analysis of core and accessory genomic regions. BMC Bioinformatics 11:461. DOI: 10.1186/1471-2105-11-461.

Larsen MV., Cosentino S., Rasmussen S., Friis C., Hasman H., Marvig RL., Jelsbak L., Sicheritz-Ponten T., Ussery DW., Aarestrup FM., Lund O. 2012. Multilocus Sequence 
946

947

948

949

950

951

952

953

954

955

956

957

958

959

960

961

962

963

964

965

966

967

968

969

970

971

Typing of Total-Genome-Sequenced Bacteria. Journal of Clinical Microbiology 50:13551361. DOI: $10.1128 / \mathrm{jcm} .06094-11$.

Lefort V., Desper R., Gascuel O. 2015. FastME 2.0: A Comprehensive, Accurate, and Fast Distance-Based Phylogeny Inference Program: Table 1. Molecular Biology and Evolution 32:2798-2800. DOI: 10.1093/molbev/msv150.

Lemoine F., Correia D., Lefort V., Doppelt-Azeroual O., Mareuil F., Cohen-Boulakia S., Gascuel O. 2019. NGPhylogeny.fr: new generation phylogenetic services for nonspecialists. Nucleic Acids Research 47. DOI: 10.1093/nar/gkz303.

Letunic I., Bork P. 2019. Interactive Tree Of Life (iTOL) v4: recent updates and new developments. Nucleic Acids Research 47. DOI: 10.1093/nar/gkz239.

Lim S., Lee D-H., Kwak W., Shin H., Ku H-J., Lee J-E., Lee GE., Kim H., Choi S-H., Ryu S., Lee J-H. 2015. Comparative Genomic Analysis of Staphylococcus aureus FORC_001 and S. aureus MRSA252 Reveals the Characteristics of Antibiotic Resistance and Virulence Factors for Human Infection. Journal of Microbiology and Biotechnology 25:98-108. DOI: 10.4014/jmb.1410.10005.

Lina G., Piemont Y., Godail-Gamot F., Bes M., Peter M-O., Gauduchon V., Vandenesch F., Etienne J. 1999. Involvement of Panton-Valentine Leukocidin--Producing Staphylococcus aureus in Primary Skin Infections and Pneumonia. Clinical Infectious Diseases 29:11281132. DOI: $10.1086 / 313461$.

Lindsay JA. 2010. Genomic variation and evolution of Staphylococcus aureus. International Journal of Medical Microbiology 300:98-103. DOI: 10.1016/j.ijmm.2009.08.013.

Lindsay JA. 2014. Staphylococcus aureus genomics and the impact of horizontal gene transfer. International Journal of Medical Microbiology 304:103-109. DOI: 10.1016/j.ijmm.2013.11.010.

Lindsay JA., Moore CE., Day NP., Peacock SJ., Witney AA., Stabler RA., Husain SE., Butcher PD., Hinds J. 2006. Microarrays Reveal that Each of the Ten Dominant Lineages of 
972

973

974

975

976

977

978

979

980

981

982

983

984

985

986

987

988

989

990

991

992

993

994

995

996

Staphylococcus aureus Has a Unique Combination of Surface-Associated and Regulatory Genes. Journal of Bacteriology 188:669-676. DOI: 10.1128/jb.188.2.669-676.2006.

Lister JL., Horswill AR. 2014. Staphylococcus aureus biofilms: recent developments in biofilm dispersal. Frontiers in Cellular and Infection Microbiology 4. DOI: 10.3389/fcimb.2014.00178.

Liu B., Zheng D., Jin Q., Chen L., Yang J. 2018. VFDB 2019: a comparative pathogenomic platform with an interactive web interface. Nucleic Acids Research 47. DOI: 10.1093/nar/gky1080.

Maddux M.S. 1991. Effects of ß-lactamase-mediated antimicrobial resistance: the role of ßlactamase inhibitors. Journal of human pharmacology and drug therapy 11:40S-50S.

Maurer LM., Yohannes E., Bondurant SS., Radmacher M., Slonczewski JL. 2005. pH Regulates Genes for Flagellar Motility, Catabolism, and Oxidative Stress in Escherichia coli K-12. Journal of Bacteriology 187:304-319. DOI: 10.1128/jb.187.1.304-319.2005.

Mazmanian SK. 1999. Staphylococcus aureus Sortase, an Enzyme that Anchors Surface Proteins to the Cell Wall. Science 285:760-763. DOI: 10.1126/science.285.5428.760.

McCarthy AJ., Lindsay JA. 2012. The distribution of plasmids that carry virulence and resistance genes in Staphylococcus aureus is lineage associated. BMC Microbiology 12:104. DOI: 10.1186/1471-2180-12-104.

McCarthy AJ., Loeffler A., Witney AA., Gould KA., Lloyd DH., Lindsay JA. 2014. Extensive Horizontal Gene Transfer during Staphylococcus aureus Co-colonization In Vivo. Genome Biology and Evolution 6:2697-2708. DOI: 10.1093/gbe/evu214.

McNair K., Bailey BA., Edwards RA. 2012. PHACTS, a computational approach to classifying the lifestyle of phages. Bioinformatics 28:614-618. DOI: 10.1093/bioinformatics/bts014.

Medini D, Donati C, Tettelin H, Masignani V, Rappuoli R. 2005. The microbial pan-genome. Current Opinion in Genetic Development 15: 589-594 
997 Meier-Kolthoff JP., Auch AF., Klenk H-P., Göker M. 2013. Genome sequence-based species 998 delimitation with confidence intervals and improved distance functions. BMC 999 Bioinformatics 14:60. DOI: 10.1186/1471-2105-14-60.

1000

1001

1002

1003

1004

1005

1006

1007

1008

1009

1010

1011

1012

1013

1014

1015

1016

1017

1018

1019

1020

1021

1022

Meier-Kolthoff JP., Göker M. 2019. TYGS is an automated high-throughput platform for stateof-the-art genome-based taxonomy. Nature Communications 10. DOI: 10.1038/s41467019-10210-3.

Melish ME., Glasgow LA. 1970. The Staphylococcal Scalded-Skin Syndrome. New England Journal of Medicine 282:1114-1119. DOI: 10.1056/nejm197005142822002.

Mistry H., Sharma P., Mahato S., Saravanan R., Kumar PA., Bhandari V. 2016. Prevalence and Characterization of Oxacillin Susceptible mecA-Positive Clinical Isolates of Staphylococcus aureus Causing Bovine Mastitis in India. Plos One 11. DOI: 10.1371/journal.pone.0162256.

Monecke S., Coombs G., Shore AC., Coleman DC., Akpaka P., Borg M., Chow H., Ip M., Jatzwauk L., Jonas D., Kadlec K., Kearns A., Laurent F., O'brien FG., Pearson J., Ruppelt A., Schwarz S., Scicluna E., Slickers P., Tan H-L., Weber S., Ehricht R. 2011. A Field Guide to Pandemic, Epidemic and Sporadic Clones of Methicillin-Resistant Staphylococcus aureus. PLoS ONE 6. DOI: 10.1371/journal.pone.0017936.

Morens DM., Fauci AS. 2013. Emerging Infectious Diseases: Threats to Human Health and Global Stability. PLoS Pathogens 9. DOI: 10.1371/journal.ppat.1003467.

Møretrø T., Hermansen L., Holck AL., Sidhu MS., Rudi K., Langsrud S. 2003. Biofilm Formation and the Presence of the Intercellular Adhesion Locus ica among Staphylococci from Food and Food Processing Environments. Applied and Environmental Microbiology 69:5648-5655. DOI: 10.1128/aem.69.9.5648-5655.2003.

Mottola C., Matias CS., Mendes JJ., Melo-Cristino J., Tavares L., Cavaco-Silva P., Oliveira M. 2016. Susceptibility patterns of Staphylococcus aureus biofilms in diabetic foot infections. BMC Microbiology 16. DOI: 10.1186/s12866-016-0737-0. 
1023 Naorem RS., Urban P., Goswami G., Fekete C. 2020. Characterization of methicillin-resistant Staphylococcus aureus through genomics approach. 3Biotech.

1025

1026

1027

1028

1029

1030

1031

1032

1033

1034

1035

1036

1037

1038

1039

1040

1041

1042

1043

1044

1045

1046

1047

Nasr RA., Abushady HM., Hussein HS. 2012. Biofilm formation and presence of icaAD gene in clinical isolates of staphylococci. Egyptian Journal of Medical Human Genetics 13:269274. DOI: $10.1016 /$ j.ejmhg.2012.04.007.

Nübel U., Dordel J., Kurt K., Strommenger B., Westh H., Shukla SK., Žemličková H., Leblois R., Wirth T., Jombart T., Balloux F., Witte W. 2010. A Timescale for Evolution, Population Expansion, and Spatial Spread of an Emerging Clone of Methicillin-Resistant Staphylococcus aureus. PLoS Pathogens 6. DOI: 10.1371/journal.ppat.1000855.

Nubel U., Roumagnac P., Feldkamp M., Song J-H., Ko KS., Huang Y-C., Coombs G., Ip M., Westh H., Skov R., Struelens MJ., Goering RV., Strommenger B., Weller A., Witte W., Achtman M. 2008. Frequent emergence and limited geographic dispersal of methicillinresistant Staphylococcus aureus. Proceedings of the National Academy of Sciences 105:14130-14135. DOI: 10.1073/pnas.0804178105.

Nurk S., Bankevich A., Antipov D., Gurevich AA., Korobeynikov A., Lapidus A., Prjibelski AD., Pyshkin A., Sirotkin A., Sirotkin Y., Stepanauskas R., Clingenpeel SR., Woyke T., Mclean JS., Lasken R., Tesler G., Alekseyev MA., Pevzner PA. 2013. Assembling SingleCell Genomes and Mini-Metagenomes From Chimeric MDA Products. Journal of Computational Biology 20:714-737. DOI: 10.1089/cmb.2013.0084.

O'Gara JP. 2007. ica and beyond: biofilm mechanisms and regulation in Staphylococcus epidermidis and Staphylococcus aureus. FEMS Microbiology Letters 270:179-188. DOI: 10.1111/j.1574-6968.2007.00688.x.

Otto M. 2008. Staphylococcal Biofilms. Current Topics in Microbiology and Immunology Bacterial Biofilms:207-228. DOI: 10.1007/978-3-540-75418-3_10.

Otto, M. 2014. Staphylococcus aureus toxins. Current Opinion in Microbiology. 17: 32-37 
1048 Overbeek R., Olson R., Pusch GD., Olsen GJ., Davis JJ., Disz T., Edwards RA., Gerdes S., 1049 Parrello B., Shukla M., Vonstein V., Wattam AR., Xia F., Stevens R. 2013. The SEED and 1050 the Rapid Annotation of microbial genomes using Subsystems Technology (RAST). Nucleic Acids Research 42. DOI: 10.1093/nar/gkt1226.

1052

1053

1054

1055

1056

1057

1058

1059

1060

1061

1062

1063

1064

1065

1066

1067

1068

1069

1070

1071

1072

Ozer EA. 2018. ClustAGE: A tool for clustering and distribution analysis of bacterial accessory genomic elements. BMC Bioinformatics 19:150

Payne DE., Boles BR. 2015. Emerging interactions between matrix components during biofilm development. Current Genetics 62:137-141. DOI: 10.1007/s00294-015-0527-5.

Periasamy S., Joo H-S., Duong AC., Bach T-HL., Tan VY., Chatterjee SS., Cheung GYC., Otto M. 2012. How Staphylococcus aureus biofilms develop their characteristic structure. Proceedings of the National Academy of Sciences 109:1281-1286. DOI: 10.1073/pnas.1115006109.

Pugazhendhi A., Michael D., Prakash D., Krishnamaurthy PP., Shanmuganathan R., Al-Dhabi NA., Duraipandiyan V., Arasu MV., Kaliannan T. 2020. Antibiogram and plasmid profiling of beta-lactamase producing multi drug resistant Staphylococcus aureus isolated from poultry litter. Journal of King Saud University - Science. DOI: 10.1016/j.jksus.2020.06.007.

Reffuveille F., Josse J., Vallé Q., Mongaret C., Gangloff SC. 2017. Staphylococcus aureus Biofilms and their Impact on the Medical Field. The Rise of Virulence and Antibiotic Resistance in Staphylococcus aureus. DOI: 10.5772/66380.

Richter M., Rosselló-Móra R., Glöckner FO., Peplies J. 2015. JSpeciesWS: a web server for prokaryotic species circumscription based on pairwise genome comparison. Bioinformatics 32:929-931. DOI: 10.1093/bioinformatics/btv681.

Sau S., Bhasin N., Wann ER., Lee JC., Foster TJ., Lee CY. 1997. The Staphylococcus aureus allelic genetic loci for serotype 5 and 8 capsule expression contain the type-specific genes 
1073

1074

1075

1076

1077

1078

1079

1080

1081

1082

1083

1084

1085

1086

1087

1088

1089

1090

1091

1092

1093

1094

1095

1096

1097

1098

flanked by common genes. Microbiology 143:2395-2405. DOI: 10.1099/00221287-143-72395.

Schmidt, H., \& Hensel, M. 2004. Pathogenicity islands in bacterial pathogenesis. Clinical microbiology reviews, 17(1), 14-56. https://doi.org/10.1128/cmr.17.1.14-56.2004

Sharma S., Chaudhry V., Kumar S., Patil PB. 2018. Phylogenomic Based Comparative Studies on Indian and American Commensal Staphylococcus epidermidis Isolates. Frontiers in Microbiology 9. DOI: 10.3389/fmicb.2018.00333.

Shin K., Yun Y., Yi S., Lee HG., Cho J-C., Suh K-D., Lee J., Park J. 2013. Biofilm-forming ability of Staphylococcus aureus strains isolated from human skin. Journal of Dermatological Science 71:130-137. DOI: 10.1016/j.jdermsci.2013.04.004.

Shopsin B., Mathema B., Alcabes P., Said-Salim B., Lina G., Matsuka A., Martinez J., Kreiswirth BN. 2003. Prevalence of agr Specificity Groups among Staphylococcus aureus Strains Colonizing Children and Their Guardians. Journal of Clinical Microbiology 41:456-459. DOI: 10.1128/jcm.41.1.456-459.2003.

Shukla SK., Pantrang M., Stahl B., Briska AM., Stemper ME., Wagner TK., Zentz EB., Callister SM., Lovrich SD., Henkhaus JK., Dykes CW. 2012. Comparative Whole-Genome Mapping To Determine Staphylococcus aureus Genome Size, Virulence Motifs, and Clonality. Journal of Clinical Microbiology 50:3526-3533. DOI: 10.1128/jcm.01168-12.

Singh T., Bhutia K., Adhikari L., Biswas S. 2015. Molecular characterization of community- \& hospital-acquired methicillin-resistant \& methicillin-sensitive Staphylococcus aureus isolates in Sikkim. Indian Journal of Medical Research 142:330. DOI: 10.4103/09715916.166600 .

Sugimoto S., Iwamoto T., Takada K., Okuda K-I., Tajima A., Iwase T., Mizunoe Y. 2013. Staphylococcus epidermidis Esp Degrades Specific Proteins Associated with Staphylococcus aureus Biofilm Formation and Host-Pathogen Interaction. Journal of Bacteriology 195:1645-1655. DOI: 10.1128/jb.01672-12. 
1099 Sullivan, M. J., Petty, N. K., \& Beatson, S. A. (2011). Easyfig: a genome comparison

1100

1101

1102

1103

1104

1105

1106

1107

1108

1109

1110

1111

1112

1113

1114

1115

1116

1117

1118

1119

1120

1121

1122

1123 visualizer. Bioinformatics (Oxford, England), 27(7), 1009-1010. https://doi.org/10.1093/bioinformatics/btr039.

Tchoupa AK., Watkins KE., Jones RA., Kuroki A., Alam MT., Perrier S., Chen Y., Unnikrishnan M. 2019. The type VII secretion system protects Staphylococcus aureus against antimicrobial host fatty acids. DOI: 10.1101/572172.

Tettelin H., Riley D., Cattuto C., Medini D. 2008. Comparative genomics: the bacterial pangenome. Current Opinion in Microbiology 11:472-477. DOI: 10.1016/j.mib.2008.09.006.

Valenzuela M., Cerda O., Toledo H. 2003. Overview on chemotaxis and acid resistance in Helicobacter pylori. Biological Research 36. DOI: 10.4067/s0716-97602003000300014.

Van Heel AJ., De Jong A., Song C., Viel JH., Kok J., Kuipers OP. 2018. BAGEL4: a userfriendly web server to thoroughly mine RiPPs and bacteriocins. Nucleic Acids Research 46. DOI: $10.1093 /$ nar/gky383.

Vandenesch F., Naimi T., Enright MC., Lina G., Nimmo GR., Heffernan H., Liassine N., Bes M., Greenland T., Reverdy M-E., Etienne J. 2003. Community-Acquired MethicillinResistantStaphylococcus aureusCarrying Panton-Valentine Leukocidin Genes: Worldwide Emergence. Emerging Infectious Diseases 9:978-984. DOI: 10.3201/eid0908.030089.

Vogel V., Falquet L., Calderon-Copete SP., Basset P., Blanc DS. 2012. Short Term Evolution of a Highly Transmissible Methicillin-Resistant Staphylococcus aureus Clone (ST228) in a Tertiary Care Hospital. PLoS ONE 7. DOI: 10.1371/journal.pone.0038969.

Wamel WJBV., Rooijakkers SHM., Ruyken M., Kessel KPMV., Strijp JAGV. 2006. The Innate Immune Modulators Staphylococcal Complement Inhibitor and Chemotaxis Inhibitory Protein of Staphylococcus aureus Are Located on $\beta$-Hemolysin-Converting Bacteriophages. Journal of Bacteriology 188:1310-1315. DOI: 10.1128/jb.188.4.13101315.2006. 
1124 Wattam AR., Abraham D., Dalay O., Disz TL., Driscoll T., Gabbard JL., Gillespie JJ., Gough R., 1125 Hix D., Kenyon R., Machi D., Mao C., Nordberg EK., Olson R., Overbeek R., Pusch GD., 1126 Shukla M., Schulman J., Stevens RL., Sullivan DE., Vonstein V., Warren A., Will R., 1127 Wilson MJ., Yoo HS., Zhang C., Zhang Y., Sobral BW. 2013. PATRIC, the bacterial 1128 bioinformatics database and analysis resource. Nucleic Acids Research 42. DOI: 10.1093/nar/gkt1099.

Zhang K., Mcclure J-A., Conly JM. 2012. Enhanced multiplex PCR assay for typing of staphylococcal cassette chromosome mec types I to $\mathrm{V}$ in methicillin-resistant Staphylococcus aureus. Molecular and Cellular Probes 26:218-221. DOI: 10.1016/j.mcp.2012.04.002. Factors, Biofilm Formation and Antibiotic Resistance of Staphylococcus aureus Isolates From Pork Production. Frontiers in Microbiology 9. DOI: 10.3389/fmicb.2018.01876. 


\section{Figure 1}

Comparison of linear plasmid maps by Easyfig alignment.

Coding Sequences are represented by colored arrows. Blue lines between the plasmids indicate the shared similarity regions according to BLASTn identity. CDS are characterized by functions as follows: antiseptic resistance genes (yellow), erythromycin resistance gene (light red), DNA replication (green), transposons/integrases (red), replication A gene/ hypothetical proteins/others (black), replication L gene (brown), and cadmium resistance gene (cyan). The outer scale is marked in kilobases. (A) Sequence alignment of p1G6 plasmid of SA G6 isolates with the reference pTW20_1 (FN433597.1) plasmid. (B) Sequence alignment of p2H32 plasmid of SA H32 with the reference AR_0472 (NZ_CP029648.1) plasmid. 


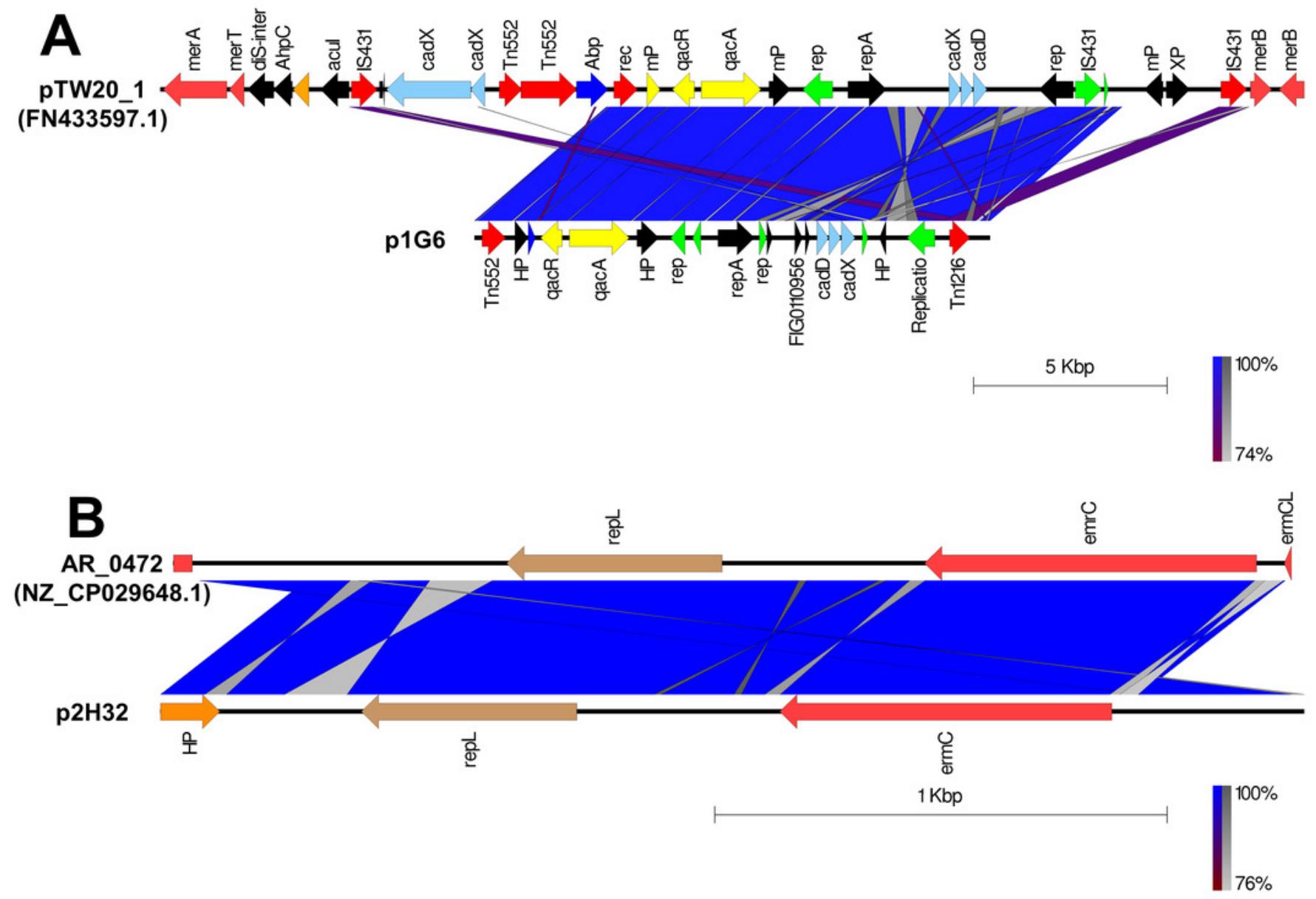




\section{Figure 2}

Heat map showing the presence (red color) and absence (blue color) of antibiotic resistance genes.

The labels on top indicate the gene names and the label on the left indicates the strains.

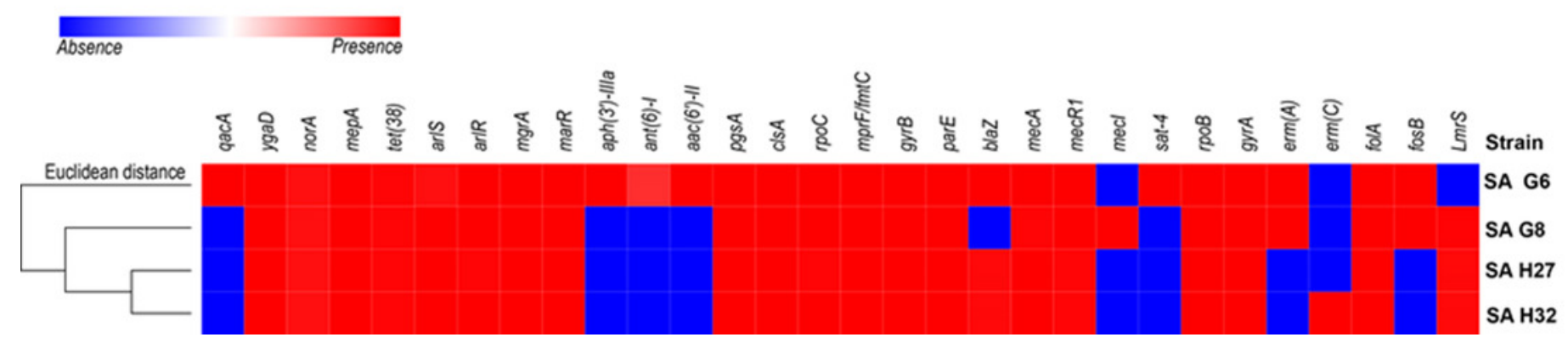




\section{Figure 3}

Heat map showing the presence (red color) and absence (blue color) of virulence factors encoding genes.

(A) Adherence and secretary factors. (B) Toxins. (C) Enzymes and antiphagocytosis (capsules) factors. The labels on top indicate the gene names and the label on the left indicates the strains.

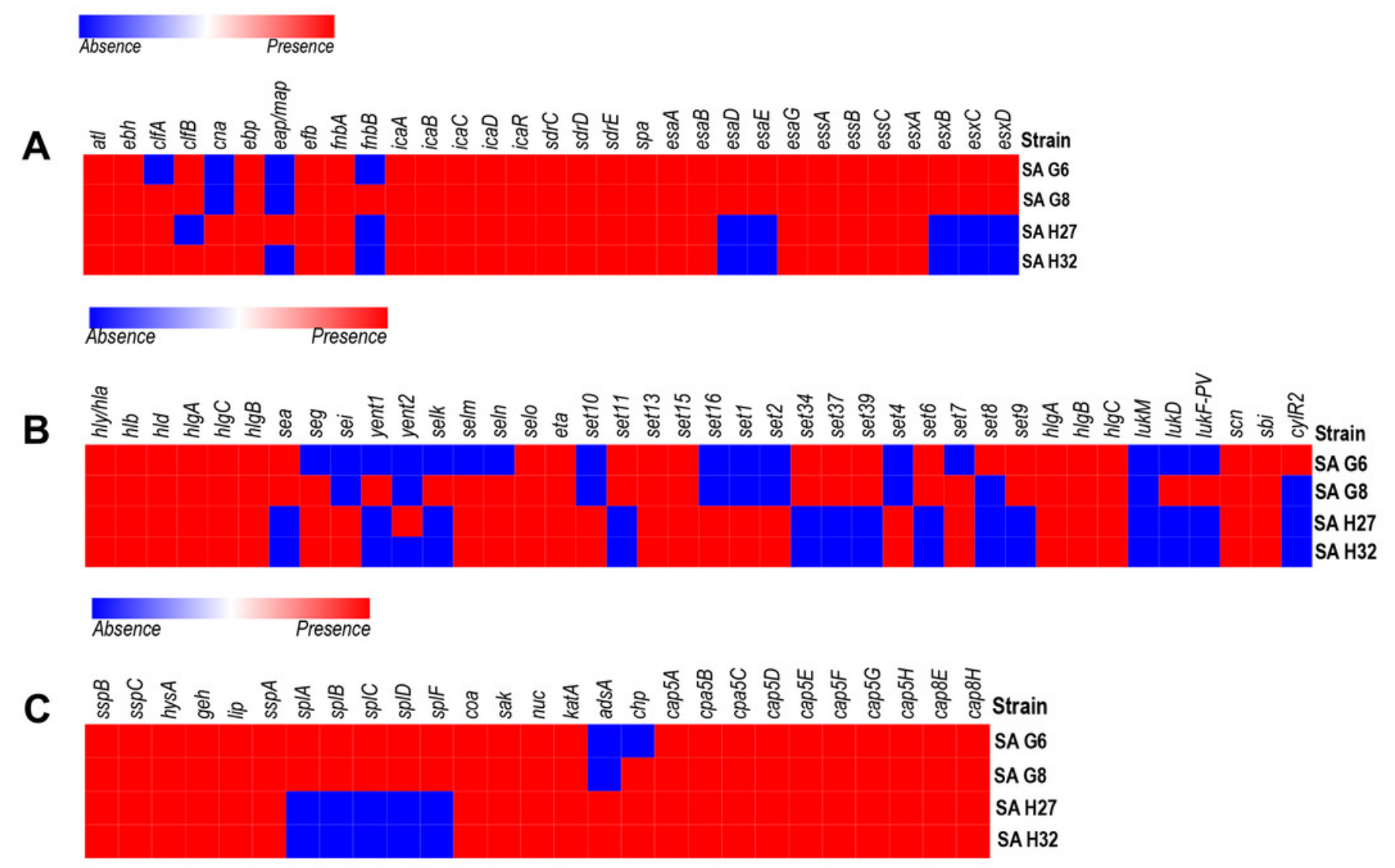


Figure 4

Circular genome comparison map showing homologous chromosome segment of four $S$. aureus genomes with the reference genome of S. aureus subsp. aureus HO 50960412 (HE681097.1) strain using CGviewer.

The inner scales designate the coordinates in kilobase pairs ( $\mathrm{kbp})$. White spaces indicate regions with no identity to the reference genome.

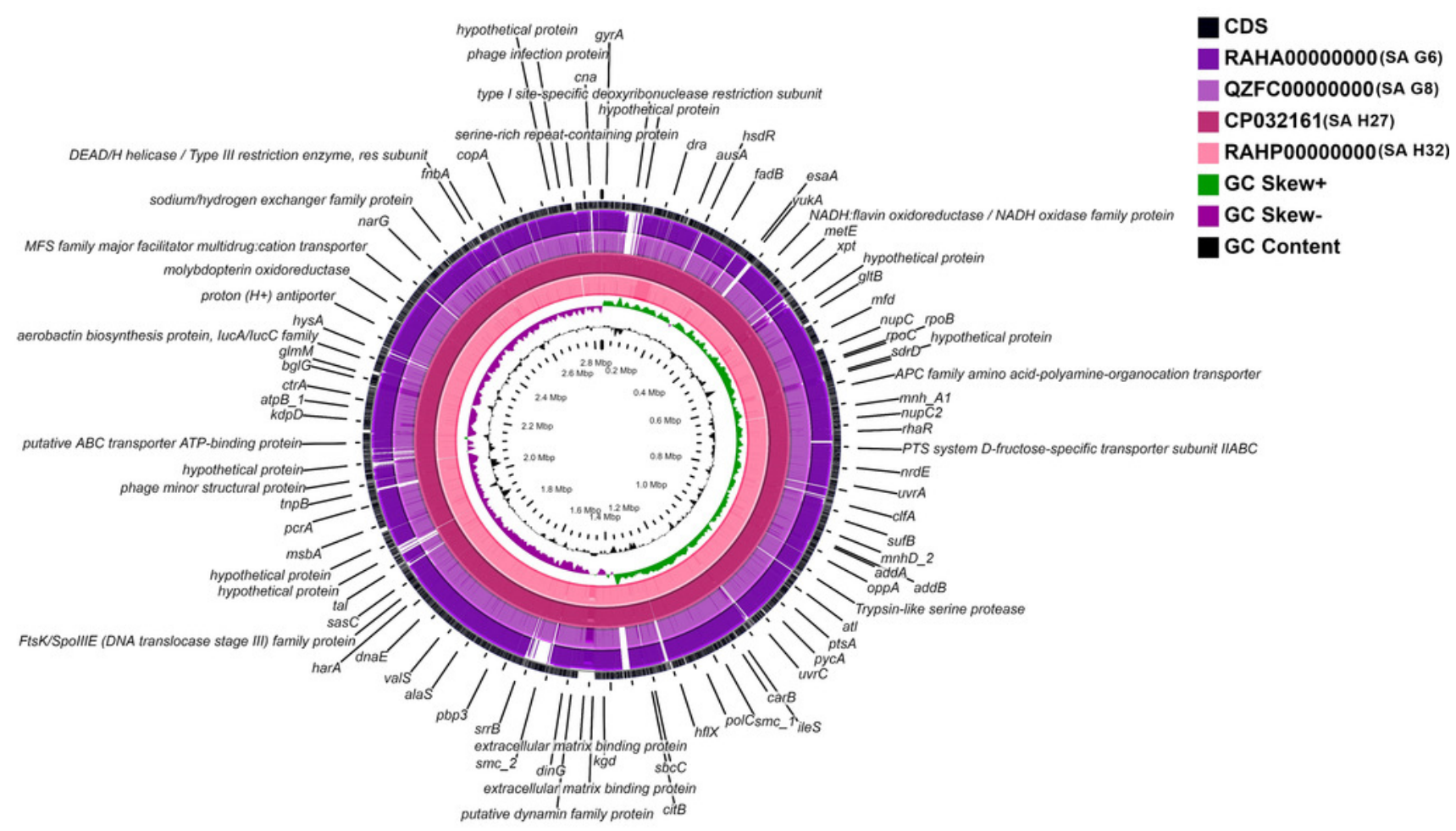


Figure 5

Comparative functional categorization of all predicted ORFs in the genomes of the $S$. aureus isolates.

(A) Percentage distribution of subsystem categories based on the SEED database. (B)

Percentage distribution of COGs based on EggNOG.
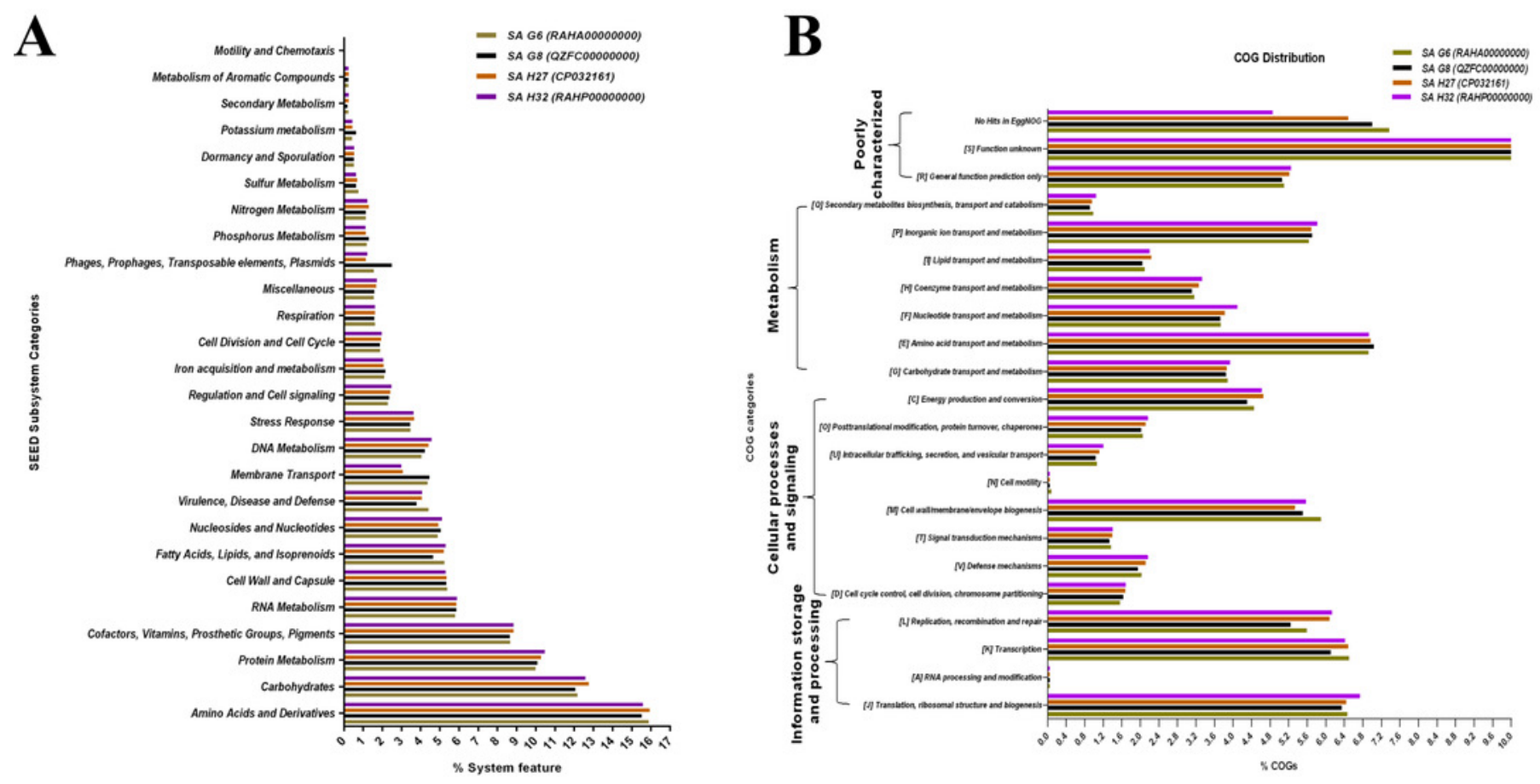


\section{Figure 6}

Pan-genome analysis of $S$. aureus strains.

(A) The Venn diagram represents the pan and core-genomes of four study genomes (SA G6, SA G8, and SA H27 and SA H32) based on orthology analysis. Overlapping regions represent common CDSs shared between the genomes. The numbers outside the overlapping regions signify the singletons of each genome. (B) Flower plot diagram representing the four study isolates and three reference strains. The core-genes of 1762 was represented in the center of the flower and the petals represent the singletons of concern genomes. (C) Core vs. pangenome plot of the seven genomes. 

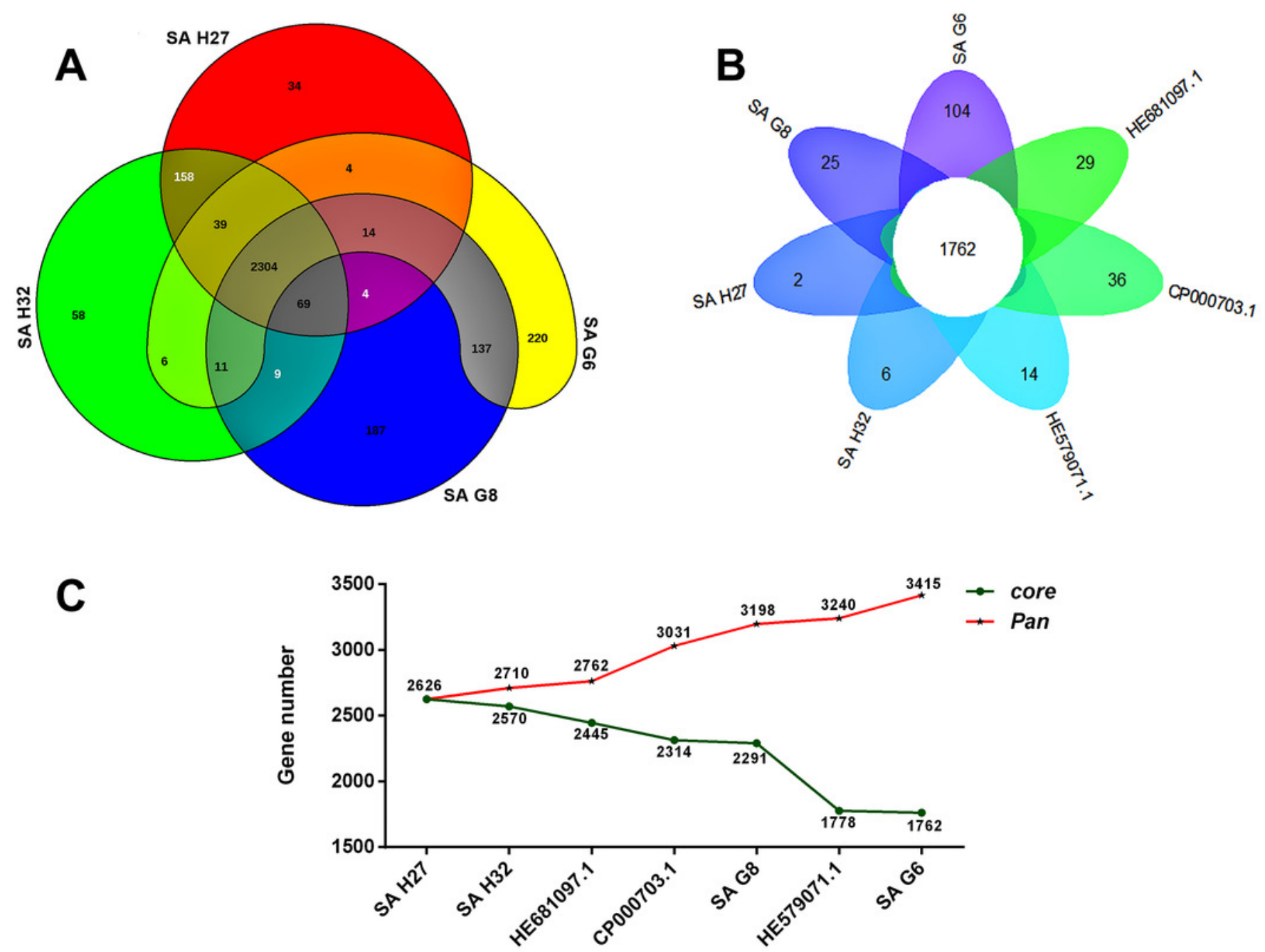

S. aureus strains 
Figure 7

Comparative phylogenetic analysis of $S$. aureus isolates strains with their closely related S. aureus strains.

Phylogenomic tree generated using closely related genome sequences. The branch lengths are scaled in terms of GBDP distance formula d5 (A), and core-genome SNP tree generated using the alignment of the high-quality SNPs and PhyML+SMS module was applied (B).

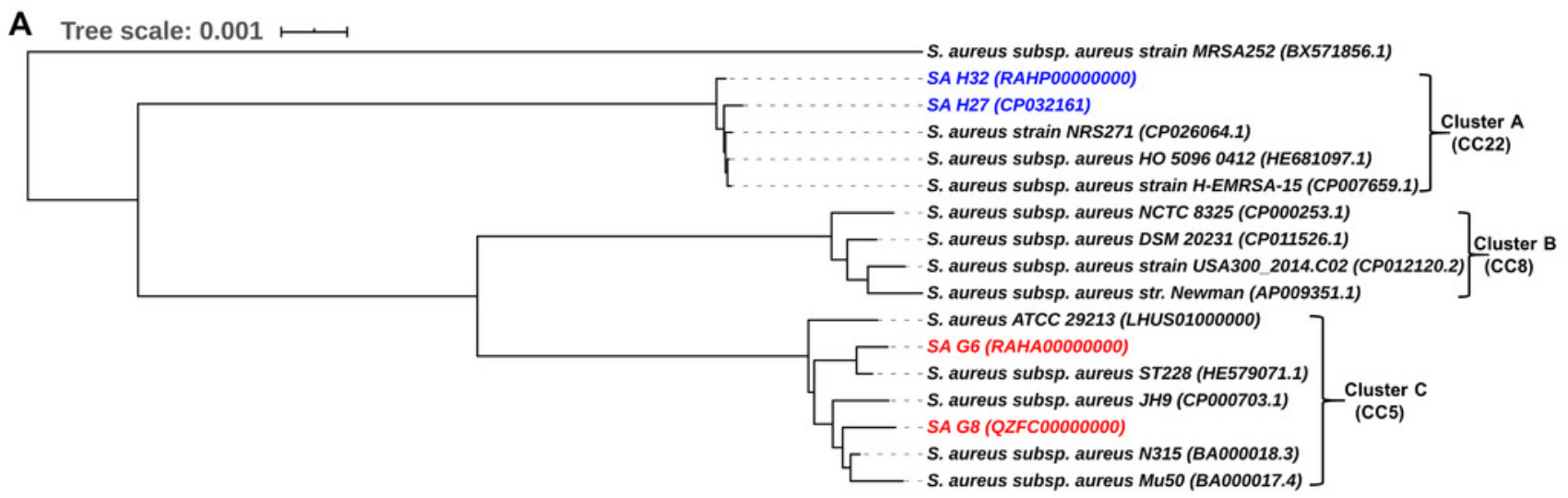

B Tree scale: 0.01 H-1

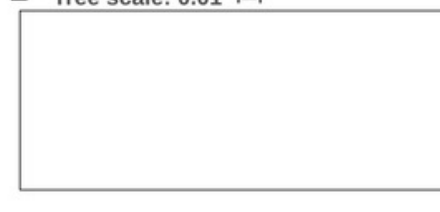

S. aureus subsp. aureus strain MRSA252 (BX571856.1)

S. aureus subsp. aureus strain H-EMRSA-15 (CP007659)

S. aureus subsp. aureus HO 50960412 (HE681097.1)

S. aureus strain NRS271 (CP02604.1)

SA H32 (RAHP00000000)

SA H27 (CP032161)

S. aureus subsp. aureus str. Newman (AP009351.1)

—S. aureus subsp. aureus USA300 (CP012120.1) Cluster B

- S. aureus subsp. aureus NCTC 8325 (CP000253.1) (CC8)

L S. aureus subsp. aureus DSM 20231 (CP011526)

S. aureus ATCC 29213 (LHUS01000000)

S. aureus subsp. aureus ST228 (HE579071.3)

SA G6 (RAHA00000000)

subsp. aureus strain Mu50 DNA (BA000017.4) Cluster C

S. aureus strain N315 (BA000018)

L SA G8 (QZFC00000000)

S. aureus subsp. aureus strain JH9 (CP00703.1) 


\section{Table $\mathbf{1}$ (on next page)}

General genomic features of $S$. aureus genomes in this study 


\section{Table 1:}

2 General genomic features of $S$. aureus genomes in this study

\begin{tabular}{|c|c|c|c|c|}
\hline Strains & SA G6 & SA G8 & SA H27 & SA H32 \\
\hline Size (bp) & 2856214 & 2857863 & 2783185 & 2786627 \\
\hline Contigs & 103 & 83 & 44 & 63 \\
\hline Scaffolds & 22 & 15 & 1 & 3 \\
\hline N50 (bp) & 125160 & 263953 & 328241 & 208577 \\
\hline GC\% & 32.79 & 32.81 & 32.73 & 32.72 \\
\hline CDS & 2734 & 2743 & 2630 & 2657 \\
\hline $\begin{array}{c}\text { Genes } \\
\text { assigned to } \\
\text { SEED }\end{array}$ & 2101 & 2169 & 2014 & 2036 \\
\hline rRNA & 9 & 10 & 8 & 9 \\
\hline tRNA & 61 & 60 & 57 & 60 \\
\hline $\begin{array}{c}\text { Prophage } \\
\text { Regions }\end{array}$ & 3 & 5 & 3 & 1 \\
\hline \#Plasmids & p1G6 & - & - & p2H32 \\
\hline $\begin{array}{c}S C C m e c \\
\text { type }\end{array}$ & I & II & IVa & IVa \\
\hline MLST & ST228 & ST225 & ST22 & ST22 \\
\hline Spa type & $\mathrm{t} 535$ & $\mathrm{t} 003$ & $\mathrm{t} 379$ & $\mathrm{t} 1258$ \\
\hline agr-type & II & II & $\mathrm{I}$ & $\mathrm{I}$ \\
\hline $\begin{array}{c}\text { Accession } \\
\text { no. }\end{array}$ & RAHA00000000 & QZFC00000000 & СР032161 & RAHP00000000 \\
\hline
\end{tabular}

3 \#Plasmids: The presence of plasmid in genome is indicated by plasmid name, while absent is

4 represented by a minus (-) sign. 